\title{
SOLAR-COLLECTOR TEST REPORT
}

Compiled by

William E. Stoney
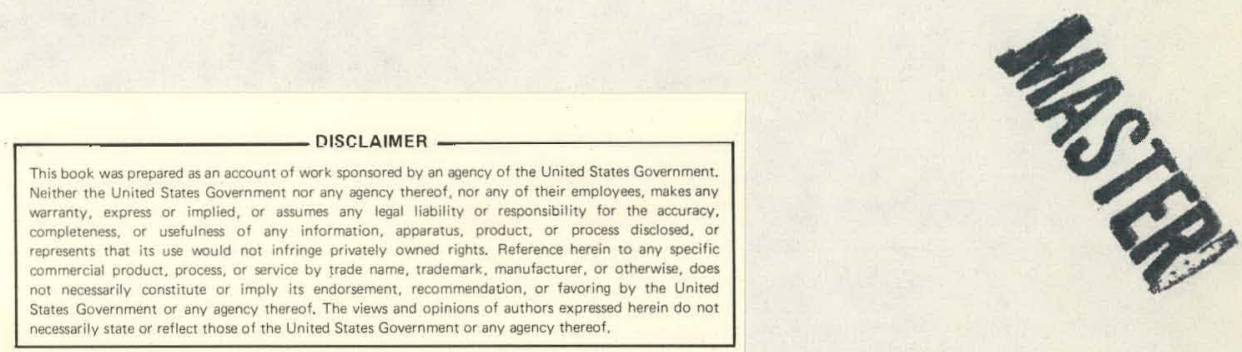

June 1981

NOTICE

PORTIONS OF THIS REPORT ARE ILIEGTBLE. It has been reproduced from the best available copy to permit the broadest possible availability.

Prepared by

Florida Solar Energy Center

$\longrightarrow$ Testing and Laboratories Division

for

4690 SOUTHERN SOLAR ENERGY CENTER

and

\section{U.S. DEPARTMENT OF ENERGY}

under contract DE-AC02-79CS30166- 


\section{DISCLAIMER}

This report was prepared as an account of work sponsored by an agency of the United States Government. Neither the United States Government nor any agency Thereof, nor any of their employees, makes any warranty, express or implied, or assumes any legal liability or responsibility for the accuracy, completeness, or usefulness of any information, apparatus, product, or process disclosed, or represents that its use would not infringe privately owned rights. Reference herein to any specific commercial product, process, or service by trade name, trademark, manufacturer, or otherwise does not necessarily constitute or imply its endorsement, recommendation, or favoring by the United States Government or any agency thereof. The views and opinions of authors expressed herein do not necessarily state or reflect those of the United States Government or any agency thereof. 


\section{DISCLAIMER}

Portions of this document may be illegible in electronic image products. Images are produced from the best available original document. 


\section{Solar Collector \\ Test Report}

Mode 7s: $x-29, x-31, \& x-32$

Brookhaven National Laboratories

Solar Technology Group, Bldg. 701

Upton, New York 11973

Florida Solar Energy Center Testing and Laboratories Division 300 State Road 401.

Cape Canaveral, Florida $32920^{\circ}$
$T \& L$

Test Report

$80150,1,2$

Approved: Aames Oraml

Test Manager

Date: 12 May 1981

Revision Date: 9 feune 1981 


\section{TABLE OF CONTENTS}

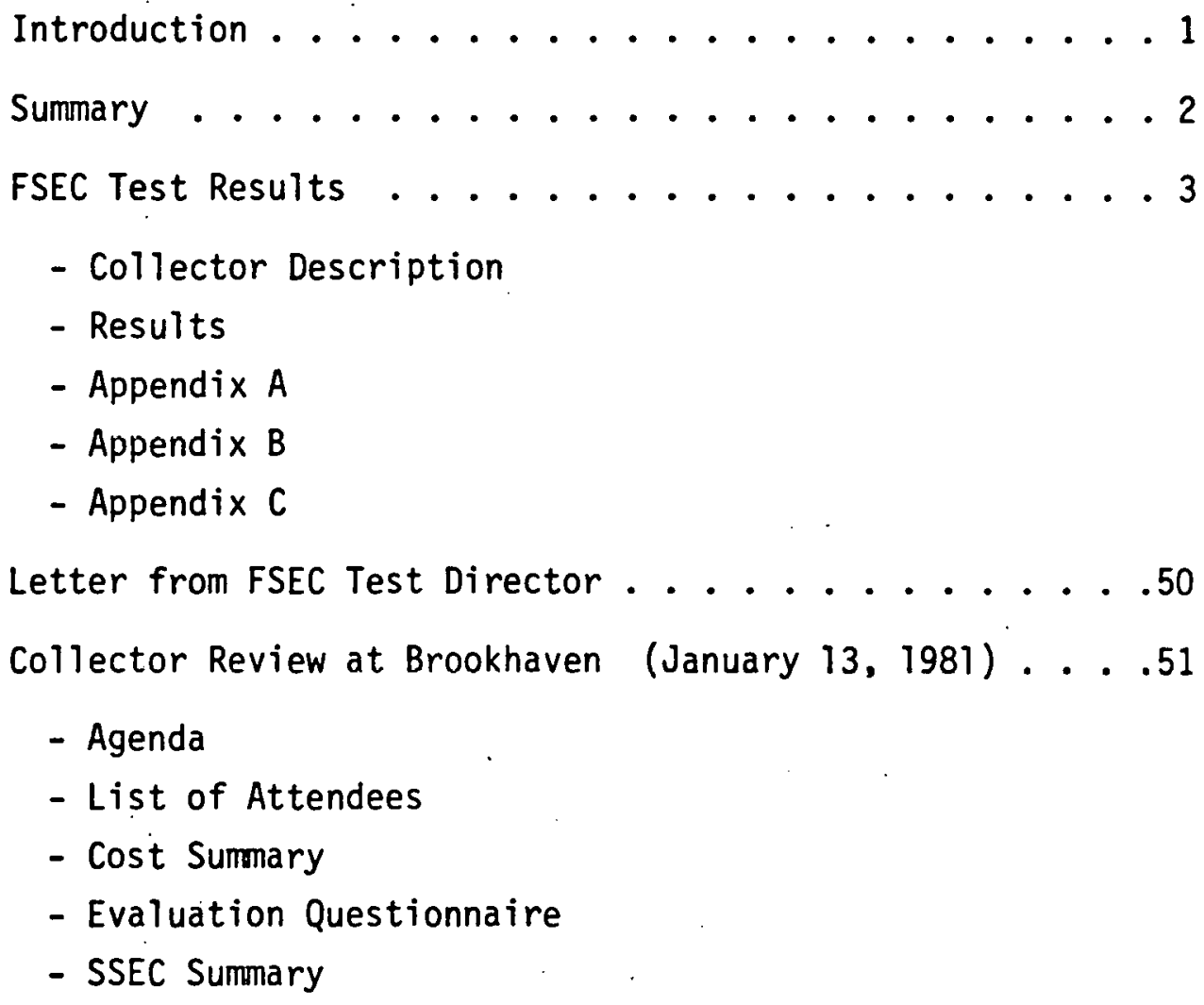

- Agenda

- List of Attendees

- Cost Summary

- Evaluation Questionnaire

- SSEC Summary 


\section{TEST REPORT CONTENTS}

1. Collector Description

1.1 Collector Identification

1.2 Collector Construction

2. Results

2.1 Introduction

2.2 Explanation of Results

Efficiency Curve

Incident Angle Modifier

Data Tabulation

2.3 Computer Printout Key

2:4 Results of Static Tests

2.5 Explanation of Efficiency Curve

2.6 Performance Test Results, English Units

Appendix A

Partial Data, Model X-29

Appendix B

Partial Data, Model X-31

Appendix C

Partial Data, Model $\mathrm{X}-32$ 
Introduction

This report summarizes activities undertaken by SSEC to evaluate the commercial readiness and potential of an all plastic solar hot water collector under development by Brookhaven Laboratories under contract to the Department of Energy. The Center's activities involved two separate functions. The first was to sponsor the testing of the collector at the Florida Solar Energy Center. The results of these tests are reported in detail in Section 1. Section 1 also includes a letter from the director of the testing and laboratories division of FSEC to Dr. Wilhelm of Brookhaven, providing a correction to part of the original report and summing up his opinions as to the merits of the collector. The second involvement was the participation by Mr. Stoney of SSEC in a review of the collector held at Brookhaven on January 13, 1981. The meeting was called by Brookhaven with the expressed purpose of obtaining an outside evaluation of the collector and recommendations as to further activities needed to bring the collector to commercial readiness. Descriptions of this meeting and SSEC's summary comments are contained in Section B of this report. 
Summary

The FSEC tests show that the Brookhaven collector compared favorably with other collectors of its type. While there were some changes noted after the exposure test, in the opinion of the test director these changes were caused by problems peculiar to the prototype construction of the test article.

In the opinion of SSEC, the review panel came to essentially unanimous conclusions. The committee agreed that the Brookhaven design demonstrated that a thin-film plastic absorber could be built to produce performance equal to, or better than the mid-range of conventional copper or aluminum absorbers. It was agreed that thin-film pastic collector production costs could probably be at least half that of currently available collectors. The committee's confidence in the performance and cost potential of the thin-film concept led them to propose that extensive efforts should be made to present their findings to those sections of industry capable of creating commercial products based on the Brookhaven concept. 
1. COLLECTOR DESCRIPTION

\subsection{Collector Identification}

Manufacturer and Address: Brookhaven National Laboratories Solar Technology Group, Bldg. 701 Upton, New York 11973

Collector Model Number: $\quad x-32$

\subsection{Collector Construction}

Overall Dimensions:

Length: $2.238 \mathrm{~m}$

Width: $\quad 0.811 \mathrm{~m}$

Depth: $0.984 \mathrm{~m}$

Overall Front:Area: (length $x$ width) 1.816 square meters

Height of outlet above inlet when collector tilt is $90^{\circ}: 2.238 \mathrm{~m}$

Glazing:

Material: Dupont Tedlar

Thickness: $0.91 \mathrm{~cm}$

Transmittance*: Not Given

No. of Cover Plates: one

Transparent Frontal Dimensions:

Length: $2.188 \mathrm{~m}$

Width: $\quad 0.760 \mathrm{~m}$

Area: $\quad 1.663 \mathrm{~m}$

Absorber:

Material: Two sheets of Tefzel Aluminum laminated together with integral fluid passages.

Length: $\quad 2.442 \mathrm{~m}$

Width: $\quad 0.760 \mathrm{~m}$

Area: $\quad 1.857$ sq. $\mathrm{m}$

Absorber coating: $99 \%$ pure nickel foil with selective surface deposits on top and high temperature silicon adhesive below

Absorptivity*: Not Given

Emissivity*: Not Given 
INSULATION:

TYPE: Polyisocyanurate

DIMENSIONS: $\quad 3.81 \mathrm{~cm}$. thick

K-FACTOR*: $\quad 0.02$ Watts/M.DEG.C (.14 BTU/HR. SQ. Ft. DEG.F/IN)

HEAT TRANSFER FLUID:

MATERIALS:

DENSITY:

Water

SPECIFIC HEAT:

977.81 KG/CUBIC Meters at 70DEG. $\dot{C}$

$4189.6 \mathrm{~J} / K G$ DEG.C. at 70DEG.C

COLLECTOR WEIGHT: $\quad 10.0 \mathrm{KG}$.

COLLECTOR WEIGHT/AREA: $5.51 \mathrm{KG} / \mathrm{SQ} . \mathrm{M}$

COLLECTOR FLUID CAPACITY: 0.6 Liters

COLLECTOR FLUID CAPACITY/AREA: 0.33 Liters/sq.m.

NORMAL OPERATING TEMPERATURE RANGE: Not Given

MANUFACTURERS RECOMMENDED FLUID FLOW RATE: 1.9 Liter/Min. (0.5 GMP)

MANUFACTUREERS RECOMMENDED MAXIMUM OPERATING PRESSURE:

0. Kilopascals (O.PSIG) - Not Pressure Tested 


\subsection{RESULTS}

\subsection{Introduction}

Three prototype solar collectors, from Brookhaven Laboratories, were submitted for testing. All three have essentially the same design except for slight variations in size. The collectors were given the following file numbers: 80150,80151 and 80152, corresponding to Brookhaven Model Numbers $X-29, X-31$ and $X-32$.

On November 9, 1980 the three Brookhaven Laboratories prototype solar collectors were partially tested for thermal performance. Five data points with fluid inlet temperature slightly above ambient and four data points at approximately $12^{\circ}$ above were taken. At this point Brookhaven requested that model numbers $X-29$ and $X-31$ be returned to Brookhaven for additional testing. Model $X-32$ was modified by adding more insulation. It remained at FSEC and underwent thermal performance test and an incident angle modifier test.

Results of the thermal performance test on model $X-32$ is presented in the body of this report in graphical and tabular form. The first graph shows the efficiency plot in both the International System of Units (SI) and English Units. The second graph presents the incident angle modifier test. Finally, the tabulated test data is presented. The English Unit tabulated data is shown in section 2.6.

The partial tests conducted on models $X-29, X-31$ and $X-32$ are included in appendices $C, D$ and $E$ respectively.

\subsection{Explanation of Results}

The Brookhaven model $X-32$ solar collector was tested for incident angle modifier and thermal performance. Results of the incident angle modifier test, conducted on 12 April 1981, are presented in graphical form. The initial performance test was conducted on 25 November 1980. 
On 29 November 1980, the collector was placed on an exposure rack for a period of 46 days. During this period there were at least thirty days on which the total daily insolation level was greater than 4730 watt-hours per square meter ( $\left.1500 \mathrm{Btu} / \mathrm{ft}^{2}\right)$ and at least one day on which there was a four-hour period with a minimum insolation level of 946 watts per square meter $\left(300 \mathrm{Btu} / \mathrm{hr}-\mathrm{ft}^{2}\right)$. During the exposure test the collector rack was adjusted to keep the angle between the incoming solar radiation and a normal to the collector surface less than five degrees at solar noon. On three of the last ten days of exposure the collector was sprayed with water to simulate rain. No thermal shock/cold fill test or static pressure leakage test were conducted:on this collector.

A second thermal performance test was conducted on 4 and 13 April 1981. The performance equations in SI units are as follows:

$$
\begin{array}{ll}
\text { INITIAL TEST } & n=68.8-590.9 \mathrm{DT} / \mathrm{I} \\
& n=68.2-518.2 \mathrm{DT} / \mathrm{I}-1041.5(\mathrm{DT} / \mathrm{I})^{2} \\
\text { RETEST } & n=71.6-678.6 \mathrm{DT} / \mathrm{I} \\
& n=70.5-559.8 \mathrm{DT} / \mathrm{I}-1713.4(\mathrm{DT} / \mathrm{I})^{2}
\end{array}
$$

After all the tests had been completed, the collector was carefully inspected. The inspection revealed some evidence of deterioration resulting from the exposure test. See Section 2.4 .4 for inspection results. 


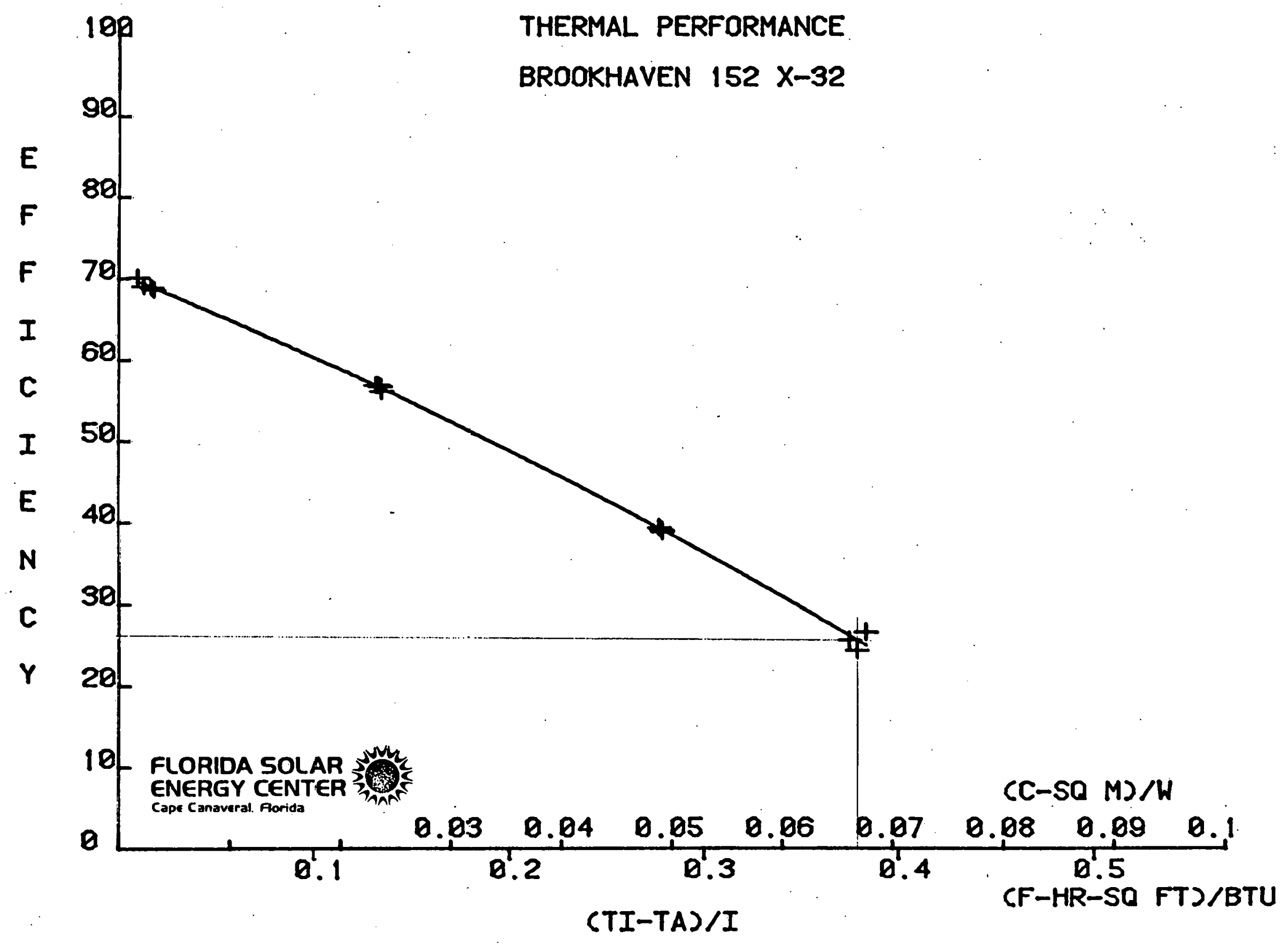




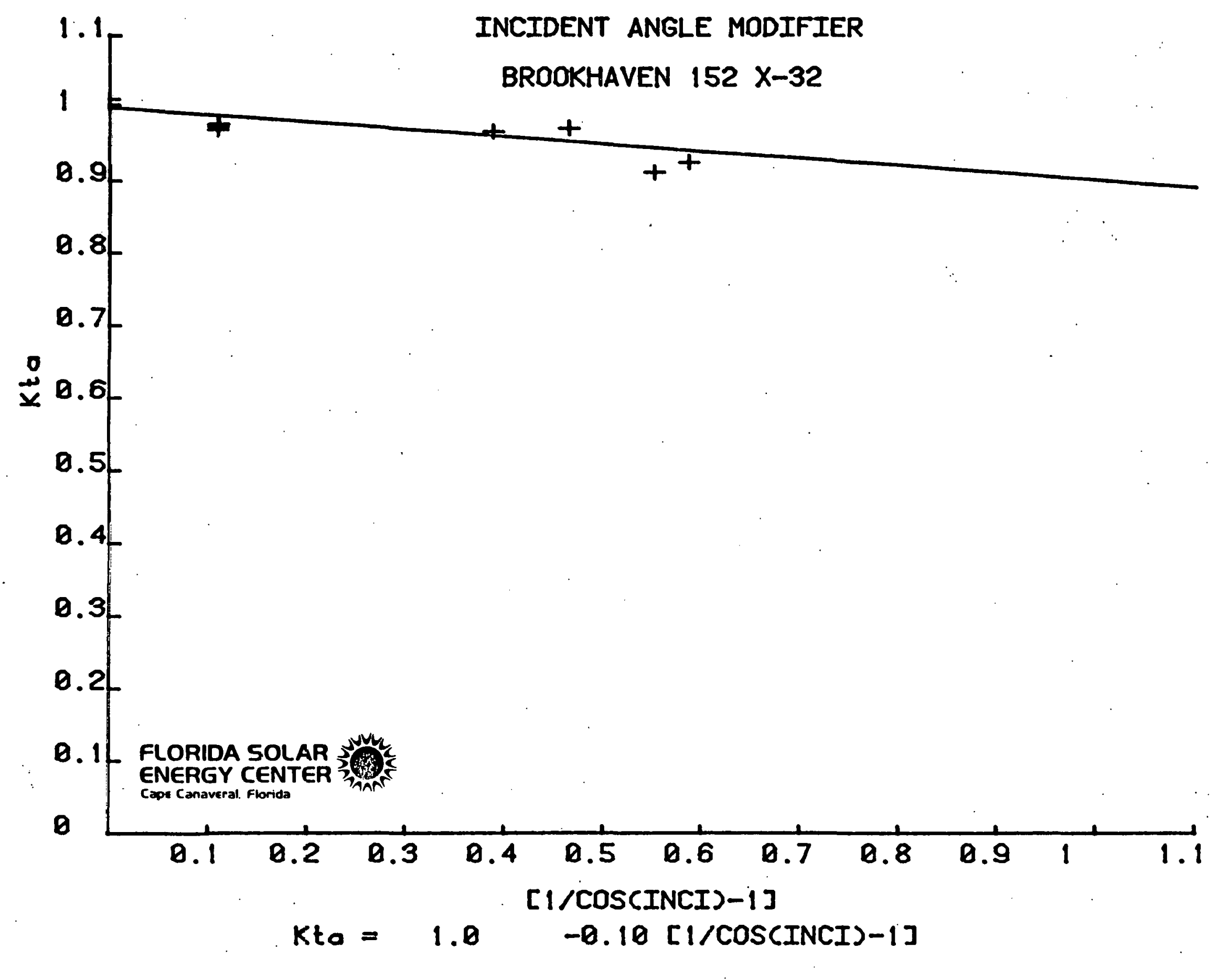




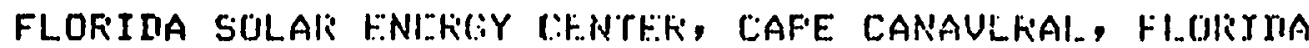

H:ITACTUKEK: BF:OOKHAUEN 1S2 FINAI. :S0152. FiF: 1 RIATE.

\section{SOLAR TIMI:} START ENI

NOTIEL: $x-3 \%$

$4 / 4 / 81$

$15: 19 \quad 15: 24$

$4 / 4 / 81$

$15: 2915: 34$

$4 / 13 / 81$

$11: 24 \quad 11: 29$

$4 / 13 / 8$

$11: 34 \quad 11: 39$

$4 / 13 / 8$

$12: 371: 2: 42$

$4 / 13 / 81 \quad 12: 42 \quad 12: 47$

$4 / 13 / 81 \quad 12: 52 \quad 12: \because 77$

$4 / 13 / 81 \quad 13: 213: 7$

$4 / 13 / 81 \quad 13: 41 \quad 13: 46$

$4 / 13 / 81 \quad 13: 46 \quad 13: 511$

$4 / 13 / 8$

$4 / 13 / 8$

$4 / 13 / 81$

$4 / 13 / 81$

$4 / 13 / 81 \quad 14: 57$ 15: 2

4/13/81 15:2 $15: 7$

$250.58 .2 \quad 37.3$

$2 ! 3.4$

25.6

$2.5 \cdot 8$

25.8

25.9

25.9

26

26.

$26.0 \quad 73.9 \quad 74.4 \quad 1.85$

$\begin{array}{llll}255.9 & 7.3 .9 & 79.3 & 1.87\end{array}$

$25.8 \quad 84.4 \quad 93$.

$25.7 \quad 89.6 .93$.

$25.3 \quad 84.7 \quad 9.3$.

$25.6 \quad 84.8 \quad 93$.

1.86

1.84

1. 85

THE UNITS WN (TI-TA)/I ARE $(C-S Q N) / W$.

$\omega$
70.3

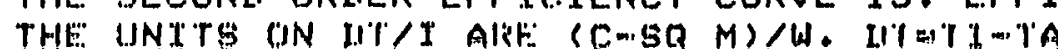

1.86

$953.6 \quad 2860 \%$

948, 4 284:51\%

$==$

71.6

$27-A P \cdot R-81$

GROSS AlitA $=1.816$ SQ $\mathrm{M}$

IE $T E F F I C \quad(T I-T A) / I$

$\begin{array}{ccc}\begin{array}{c}(T E-T I) \\ (C)\end{array} & \text { IKL } T \text { EFFIC } & (T I-T A) / I \\ C-M I N & \% & (C-S(T M) / W\end{array}$

$9.03 \quad 45.1 \cdot 68.8 \quad 0.0029$

8.89

$44.4 \quad 68.6 \quad 0.0031$

8.95

$44.7 \quad 69.0 \quad 0.002 \%$

9.11

$\begin{array}{lll}45.6 & 70.1 & 0.001 \%\end{array}$

7.51

$37.5 \quad 56.7 \quad 0.0235$

7.44

$3 \% .2$

$56.10 .0 \% 36$

$7.6 \%$

$x 8.1 \quad 56.8 \quad 0.0233$

7.68

$38.4 \quad 56.8 \quad 0.0 \% 31$

5.42

$2 \% .1 \quad 39.1 \quad 0.0184$

5.50

$27.5 \quad 39.3 \quad 0.0487$

5.49

$27.4 \quad 39.0 \quad 0.0490$

5.41

$27.2 \quad 38.8 \quad 0.0490$

3.57

$17.8 \quad 25.6 \quad 0.0660$

$3 \cdot 41$

$17.1 \quad 2.4 .3 \quad 0.0667$

$\begin{array}{llll}3.67 & 18.4 & 26.6 & 0.06 \%\end{array}$

$3.41 \quad 1 \% .1 \quad 25.0 \quad 0.0677$ $\because:=::==:======-\because=$ $-678.6(11-1 A) / 1$

$=====$
FFICIEN:

(3)

- $9.811 / 1$

$-1713.4(101 / 1) 2$ 


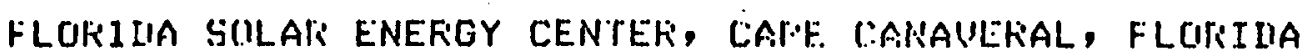

TACTUKEK: HFUOKHAVEN 152 FINAL 80152.KE 1

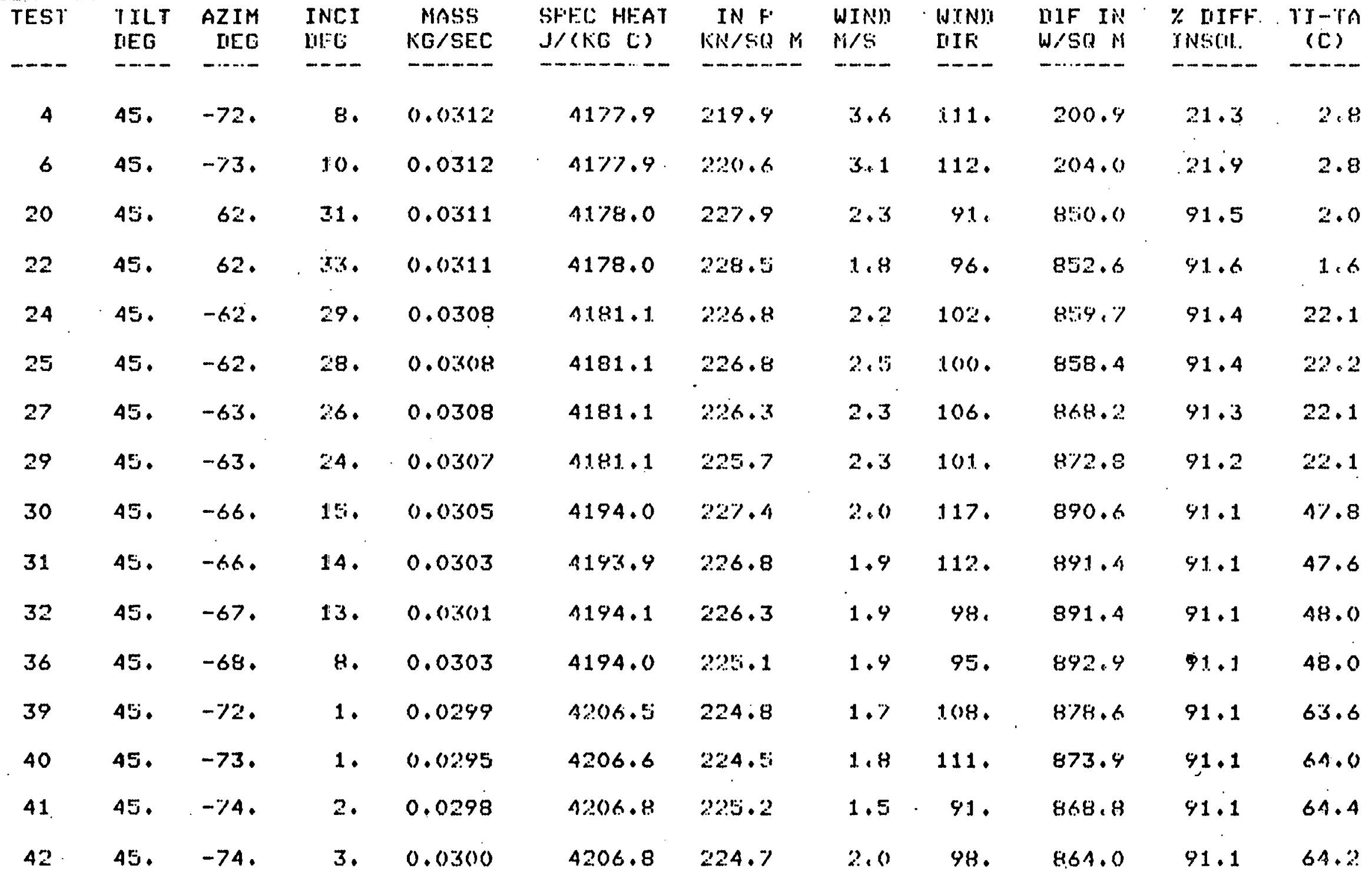

$2: 7-A \mid \cdot k i-81$

GROSS AREA $=1.816 \mathrm{sN} \mathrm{M}$
MUMI:I.: $x-32$

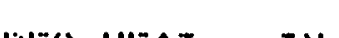




\subsection{Computer Printout Key}

TEST

DATE

SOLAR TIME

TA

TI

TE

FLOW

AVE IN

TOTAL IN

DEL T

EFFIC

$(T I-T A) / I$

TILT

AZIM

INCI

MASS

SPEC HEAT

IN $\mathrm{P}$

WIND

WIND DIR

DIF IN

\% DIFF INSOL

TI-TA

Number assigned to the data for a particular test

Date on which the test was performed

Solar time at the start and finish of the test

Ambient air temperature $\left({ }^{\circ} \mathrm{C}\right.$ or $\left.{ }^{\circ} \mathrm{F}\right)$

Temperature of the transfer fluid at the collector inlet ( ${ }^{\circ} \mathrm{C}$ or $\mathrm{OF}$ )

Temperature of the transfer fluid at the collector exit $\left({ }^{\circ} \mathrm{C}\right.$ or $\left.{ }^{\circ} \mathrm{F}\right)$

Volumetric flow rate of the transfer fluid (liters/minute or gallons/minute)

Average instantaneous insolation (watts/square meter or Btu/hour-square foot)

Total (integrated) insolation in the plane of the collector during the test period (joules/square meter or Btu/square foot)

Total (integrated) temperature rise across the collector during the test period (degree centigrade minutes or degree fahrenheit minutes)

Calculated collector efficiency for the test period (percent)

Value calculated for the abcissa of the efficiency graph $\mathrm{I}=$ average instantaneous insolation. $\left({ }^{\circ} \mathrm{C}\right.$ - square meter/ watt or OF - hour - square foot/Btu).

Angle which the collector was tilted from the horizontal (degrees)

Collector azimuth angle (degrees from due south; east positive)

Angle between the incoming radiation and a normal to the collector surface (degrees)

Mass flow rate of the transfer fluid ( $k i$ lograms/second or pounds mass/minute).

Specific heat of the transfer fluid (joules/kilogram - ${ }^{\circ} \mathrm{C}$ or Btu/pound mass - OF)

Gage pressure at the collector inlet (kiloNewtons/square meter or pounds/square inch)

Wind speed (meters/second or miles/hour)

Wind direction (degrees east from north)

Diffuse instantaneous insolation (watts/square meter or Btu/ hour-square foot)

Percent of the incoming radiation that was diffuse

Temperature difference between the inlet fluid temperature and ambient air ( ${ }^{\circ} \mathrm{C}$ or $\left.{ }^{\circ} \mathrm{F}\right)$

Note: Stars indicate that no data was taken on the indicated parameter during that test 


\subsection{Results of Static Tests}

2.4.1 Pressure Test Not Performed

Date of Test:

Static Test Pressure:

Duration of Test:

2.4.2 Pressure Drop Test Not Performed

Date of Test:

Inlet Temperature:

Flow Rate:

Inlet Pressure:

Pressure Drop:

\subsubsection{Exposure Test}

Start Date: 29 November 1980-

Completion Date: 14 January 1981

No. of Days of Exposure: 46

No. of Days of Exposure when insolation exceeded 4730 watts $/ \mathrm{m}^{2}\left(1500 \mathrm{Btu} / \mathrm{ft}^{2}\right)$ : $\quad 30$

Pressure Relief Setting: $0.0 \mathrm{kPa}(0.0 \mathrm{psig})$

Spray Tests:

Dates: $\quad 2$ January $1981 \quad 8$ January 1981 10 January 1981

Insolation: $\left.\begin{array}{c}1069 \mathrm{Watts} / \mathrm{m}^{2} \\ \left.399 \mathrm{Btu} / \mathrm{hr}-\mathrm{ft}^{2}\right)\end{array}\right)\left(\begin{array}{r}1090 \mathrm{watts} / \mathrm{m}^{2} \\ \left.346 \mathrm{Btu} / \mathrm{hr}-\mathrm{ft}^{2}\right)\end{array}\right)\left(\begin{array}{l}1040 \mathrm{watt} / \mathrm{m}^{2} \\ \left.330 \mathrm{Btu} / \mathrm{hr}^{2}-\mathrm{ft}^{2}\right)\end{array}\right.$

Thermal Shock/Cold Fill Test: Not Performed

Date:

Air Temperature:

Insolation:

Inlet Water Temperature:

Test Duration:

Flow Rate: 
Static Pressure Leakage Tests: Not Performed

Date:

Pressure:

Fluid:

Duration:

\subsubsection{Inspection Results:}

Cover Plate: The cover stretched and became distorted during exposure. Moisture appeared on the inner surface of the cover after each spray test.

Absorber Plate: See below*

Absorptive Coating: No evidence of deterioration

Collector Enclosure: No evidence of deterioration

Insulation: No evidence of deterioration

Gaskets, caulking and sealants: Foil tape around the outside peeled and separated from the collector.

Absorber Plate: Creases appeared in the absorber surface along the long axis of the collector. The creases appeared as upward projections of the absorber surface, were up to twelve in number, and were immediately above the absorber fusion lines. The crease lines, in some cases, ran nearly the entire length of the collector and in other cases ran only short distances along the fusion lines. The creases are a separation of the absorber surface/ foil laminate from the plastic layer and in some cases, a further separation of the absorber surface from the aluminum foil backing. These separations are apparently a failure of the adhesive along the absorber fusion lines. 


\subsection{Explanation of Efficiency Curve}

The test data is presented in tabular form and as an efficiency curve. The efficiency curve is based on the following equation:

$$
\eta=F_{R}(\tau \alpha)_{e}-F_{R} U_{L} \frac{\left(T_{i}-T_{a}\right)}{I}
$$

where:

$$
\begin{aligned}
n & =\text { collector efficiency } \\
F_{R} & =\text { heat removal factor } \\
(\tau \alpha)_{e} & =\text { effective transmissivity-absorptivity product } \\
U_{L} & =\text { over-all heat loss coefficient } \\
T_{i} & =\text { transfer fluid temperature at the collector inlet } \\
T_{a} & =\text { ambient air temperature } \\
I & =\text { instantaneous level of solar radiation }
\end{aligned}
$$

The standard form of the equation of a straight line is:

$$
Y=b+m x
$$

where:

$$
\begin{aligned}
& b=Y \text { axis intercept } \\
& m=\text { slope }
\end{aligned}
$$

From these two equations it can be seen that a straight line should result if a plot of efficiency versus the quantity $\frac{T_{i}-T_{a}}{I}$ is made and if the slope and intercept functions can be assumed constant. It then becomes apparent that the slope of the line is a function of the over-all heat loss coefficient and the $Y$ axis intercept is a function of the transmissivity of the cover plate(s) and the absorptivity of the absorber plate(s). In reality, however, $U_{L}$ is not constant under the test conditions since it varies with the temperature of the collector and ambient weather conditions. Therefore a second order curve is used to describe the themal perfomance of the collector. That is:

$$
Y=c+b x+a x^{2}
$$

The intercept is still related to $(\tau \alpha)_{\mathrm{e}}$ and the slope at any point on the curve is proportional to the heat loss rate for that value of $\frac{T_{i}-T_{a}}{I}$. 


\subsection{Performance Test Results, English Units}

Following is a repetition of the thermal performance curve. Although identical to the previous graphs (see Section 2.1) in content, this graph and computer printout is presented in English units. 


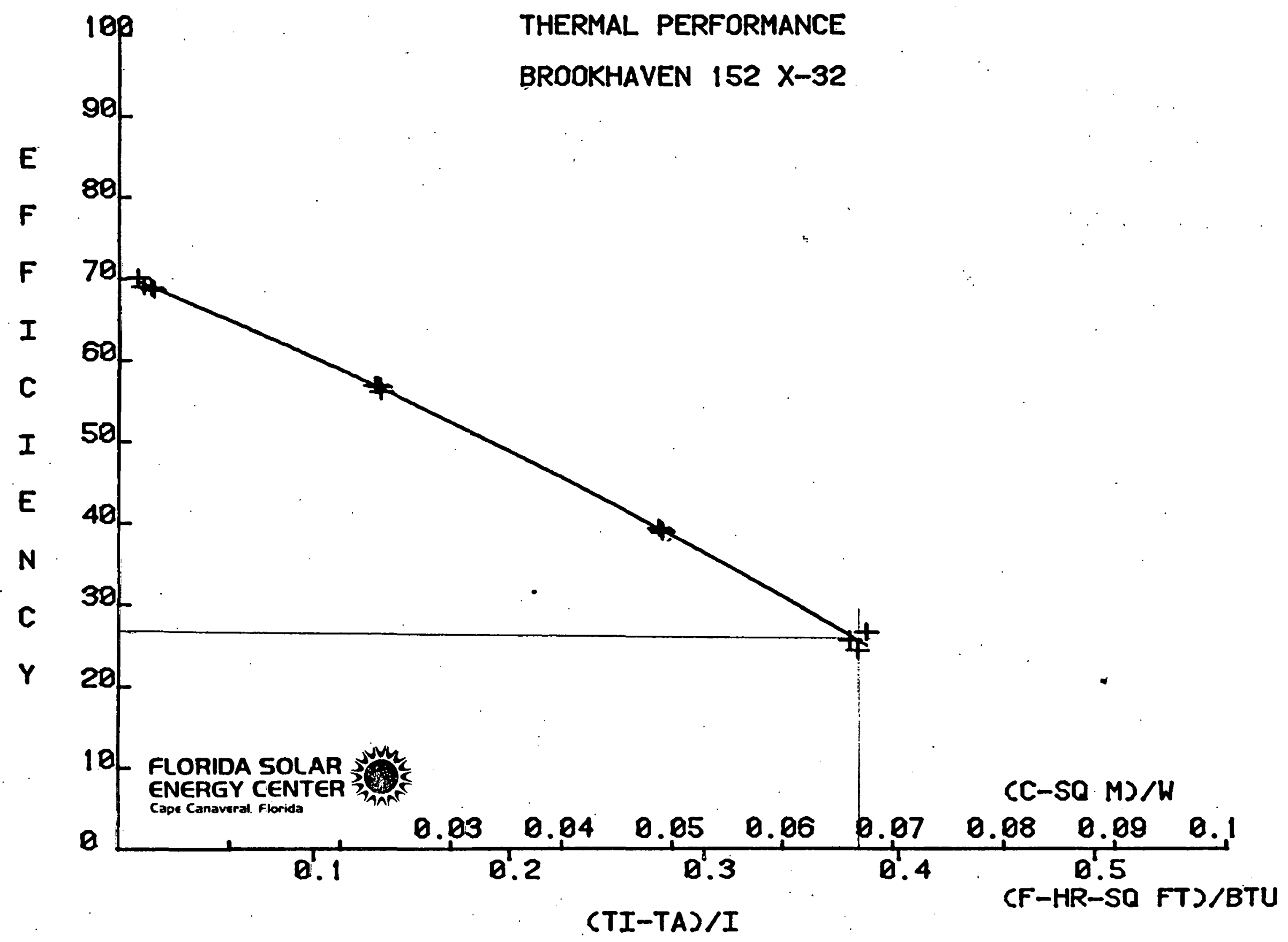




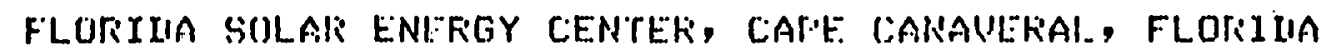

INUIFACTUREF: HKOUKHAVIN 152 FINAI. ¿ : 80152 . FEE 1 IST IIATE

-..- - - - - -

\begin{tabular}{llll} 
SOLAR TINI: TA & TI & TE \\
STAFIT ENII & (F) & (F) & (F) \\
\hline & $-\cdots$ & - & $-\cdots$
\end{tabular}

MURLi: : $x-3 \%$

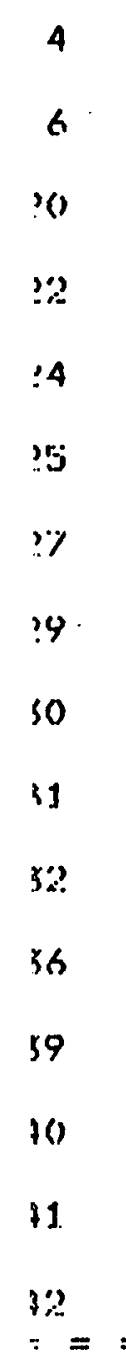

$4 / 4 / 81$

$4 / 4 / 81$

$4 / 13 / 81$

$4 / 13 / 81$

$4 / 13 / 81$

$4 / 13 / 8$

$4 / 13 / 8$

$4 / 13 / 8$

$4 / 13 / 8$

$4 / 13 / 8$

$4 / 13 / 8$

$4 / 13 / 8$

$4 / 13 / 81$

$4 / 13 / 81$

$4 / 13 / 81$

$4 / 13 / 81$

$15: 19 \quad 15: 24 \quad \% 7.9 \quad 82.8 \quad 44$.

$15: 34$

$34 \quad 77.6 \quad 42.8$

$98.8 \quad 0.498$

47.50 .496

$47.4 \quad 0.496$

$0.49 \mathrm{gi}$

131.8

0.494

$12: 42 \quad 12: 47$

$12: 52 \quad 12: 5$

\begin{abstract}
13: 2 13:
\end{abstract}
$13: 41 \quad 13: 1$

\begin{abstract}
$788.4 \quad 118$.
\end{abstract}
$3 \quad 1 \times 1$.

$7 \quad 78.6 \quad 118.4 \quad 132$.

0.494

\begin{abstract}
$78.6 \quad 118.4 \quad 13 \% .2 \quad 0.494$
\end{abstract}

\begin{abstract}
$79.1 \quad 165.1 \quad 174.9 \quad 0.49 \%$
\end{abstract}

\begin{abstract}
$\begin{array}{llllll}13: 46 \quad 13: 51 & 79.0 & 161.8 & 171.7 & 0.493\end{array}$
\end{abstract}
$3.10 .1 \quad 25.8$

$310.4 \quad 2 \pi, 4$

$3.10 .4 \quad 25.9$

\begin{abstract}
$3: 511 \quad 13: \div 66$
\end{abstract}
$78.8 \quad 165.1 \quad 175.0 \quad 0.490$

$.3 .10 \cdot 4$

\begin{abstract}
$14: 1114: 16$
\end{abstract}
$\begin{array}{llll}78.6 & 165.0 & 174.8 & 0.493\end{array}$

$311.0 \quad 25.4$

$305.9 \quad 25.5$

\begin{abstract}
$14: 4714: 5: ?$
\end{abstract}
$\begin{array}{llll}8.4 & 192.9 & 199.3 & 0.491\end{array}$

$3(1) .3 \quad 2 \times 1.4$

$3(1) .5 \quad 25.2$

$300.8 \quad 25.1$

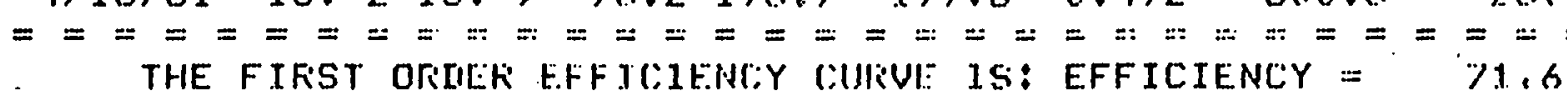
THE: UNITS ON (TI-TA)/I AliE $(F-H K-S R$ FT)/BTU. 


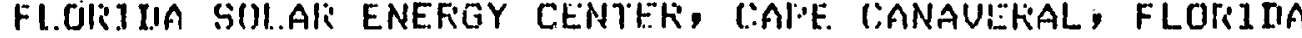

NUFACTUREK: HROOKHAUIEN 152 FINAI. I: :80152. FE 1

TILT AZIM INCI

III:Gi LRMIMIN

BTU/(LHM.F) IN

\section{$-\cdots$}

$$
20
$$$$
2
$$

$$
24
$$

$$
25
$$$$
27
$$$$
29
$$

30

- 31

$$
32
$$

\section{6}

\section{9}

40

41

42

- -.. -...

MOIIII.: $x-3 \%$

\begin{tabular}{|c|c|c|c|c|c|c|c|c|c|c|}
\hline 45. & -72 & 8. & $4 \cdot 1.3$ & 0.998 & 31.4 & 8.1 & 111. & 63.7 & $21 \cdot 3$ & $\therefore, 0$ \\
\hline 45. & -73 & 10 & 4.13 & 0.998 & $32 \%, 0$ & $\%+0$ & 112 & 64.7 & $\because 1.9$ & 5.1 \\
\hline $4: \%$. & 62. & 31. & 4.12 & 0.998 & 33.1 & $5 \cdot 1$ & 91. & 269.6 & 91.5 & 3.7 \\
\hline 45. & 62. & 33 & 4.12 & 0.998 & $3: 3.1$ & 4.1 & 96. & 270.5 & 91.6 & 2.4 \\
\hline 45. & -6.2. & 29. & 4.08 & 0.444 & $32 \%, 9$ & 5.0 & 102 & 27.79 & 91.4 & 39.8 \\
\hline 45 & -62. & 28 & 4.017 & 0.999 & $32: 9$ & 505 & $10(0)$ & 272.3 & 91.4 & 40.0 \\
\hline 45. & -63 & 26 & 4.07 & 0.999 & $3 \% .8$ & 5 & 106. & $27 \div: 4$ & 41.3 & .34 .8 \\
\hline 45. & -63. & 24 & 4.06 & 0.949 & $3 \% .7$ & 5.0 & 101. & 276,4 & 91.2 & 39.8 \\
\hline 45 & -66. & 15 & $4 .(14$ & 1.002 & 33.0 & $4 .: 3$ & 117 & $2 ! 82.5$ & $\$ 1.1$ & 86.0 \\
\hline 45. & -66. & 11 & 4.01 & 1.002 & 33.4 & $4 \cdot 3$ & 112. & 282.8 & 91.1 & $8: i, 7$ \\
\hline 45. & -67. & 13 & 3.98 & $1.00 \%$ & 32.8 & 4.3 & 98. & $28: 2.8$ & $91 \cdot 1$ & 86.3 \\
\hline 45 & -68 & 8. & $1 \cdot 01$ & 1.002 & $3 \% \cdot 6$ & 1.3 & 95. & 283.2 & $9 i .1$ & 86.4 \\
\hline 45. & -72. & 1. & 3.96 & $1.005 i$ & $3: 2 \cdot 6$ & 3.4 & 108 & 2.78 .7 & 91.1 & $114 \cdot 5$ \\
\hline $4:$ & -73 & 1. & 3.91 & $1.005 i$ & 32.6 & 4.1 & 111. & 277.2 & $91 \cdot 1$ & 115.2 \\
\hline 45. & -74 & $\therefore$ & 3.94 & 1.005 & $3: 2+7$ & 3.5 & 91. & $27: 3 \cdot 6$ & 91.1 & $115 \cdot 8$ \\
\hline $4 \because$. & -74 & 3. & 3.96 & $1 \cdot 0(0)$ & 32.6 & 4.5 & 48. & $2 \% 1, i$ & 91.1 & 115.5 \\
\hline
\end{tabular}

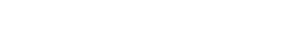

\section{$27-A F \cdot \cdot R-81$}

liRUSS AREA $=14.515 \mathrm{~F}$. 
APPENDIX A

PARTIAL DATA

MODEL $X-29$ 
1. COLLECTOR DESCRIPTION

\subsection{Collector Identification}

Manufacturer and Address:

Brookhaven National Laboratories Solar. Technology Group, Bldg. 701 Upton, Nêw York 11973

Collector Model Number: $X-29$

1.2 Collector Construction

Overall Dimensions:

$\begin{array}{lll}\text { Length: } & 2.222 & \mathrm{~m} \\ \text { Width: } & 0.816 & \mathrm{~m} \\ \text { Depth: } & 0.079 \mathrm{~m}\end{array}$

Overall Front Area: (length $\mathrm{x}$ width) $1.813 \mathrm{~m}^{2}$

Height of outlet above inlet when collector tilt is $90^{\circ}: \quad 2.222 \mathrm{~m}$ Glazing:

Material: Dupont Tedlar

Thickness: $0.01 \quad \mathrm{~cm}$

Transmittance*: Not Given

No. of Cover Plates: One

Transparent Frontal Dimensions:
Length:
2.171
m
Width:
0.816
Area:
1.771
$m^{2}$

Absorber:

Material: Two sheets of Tefzel Aluminum laminated together with Length: integral fluid passages.

Width: 2.171

$\mathrm{m}$

Area :

0.816

Absorber Coating: Absorptivity*:

$1.772 \mathrm{~m}^{2}$

Emissivity*: Not Given 
Insulation:

Type:polyị socyanurate

Dimensions: $3.81 \mathrm{~cm}$.

K-Factor $: 0.02$ watts $/ \mathrm{m}^{\circ} \mathrm{C}\left(0_{i}^{0} 14 \mathrm{Btu} / \mathrm{hr}-\mathrm{ft}^{2}-{ }^{\circ} \mathrm{F} / \mathrm{in}\right)$

Heat Transfer Fluid:

Material: Water

Density: $\quad 977.81 \mathrm{~kg} / \mathrm{m}^{3}$ at $70^{\circ} \mathrm{C}$

Specific Heat: $\quad 4189.6 \mathrm{~J} / \mathrm{kg}^{\circ} \mathrm{C}$ at $70^{\circ} \mathrm{C}$

Collector Weight: $\quad 7.0 \quad \mathrm{~kg}$

Collector Weight/Area: $3.86 \quad \mathrm{~kg} / \mathrm{m}^{2}$

Collector Fluid Capacity: $0.6 \quad$ liters

Collector Fluid Capacity/Area: $0.33 \quad 1$ iters $/ \mathrm{m}^{2}$

Normal Operating Temperature Range: Not Given

Manufacturers Recommended Fluid Flow Rate: 1.9 liter/min ( 0.5 G.PM)

Manufacturers Recommended Maximum Operating Pressure:

0.0 kilopascals ( 0.0 psig)

*Information provided by manufacturer; not verified by FSEC. 


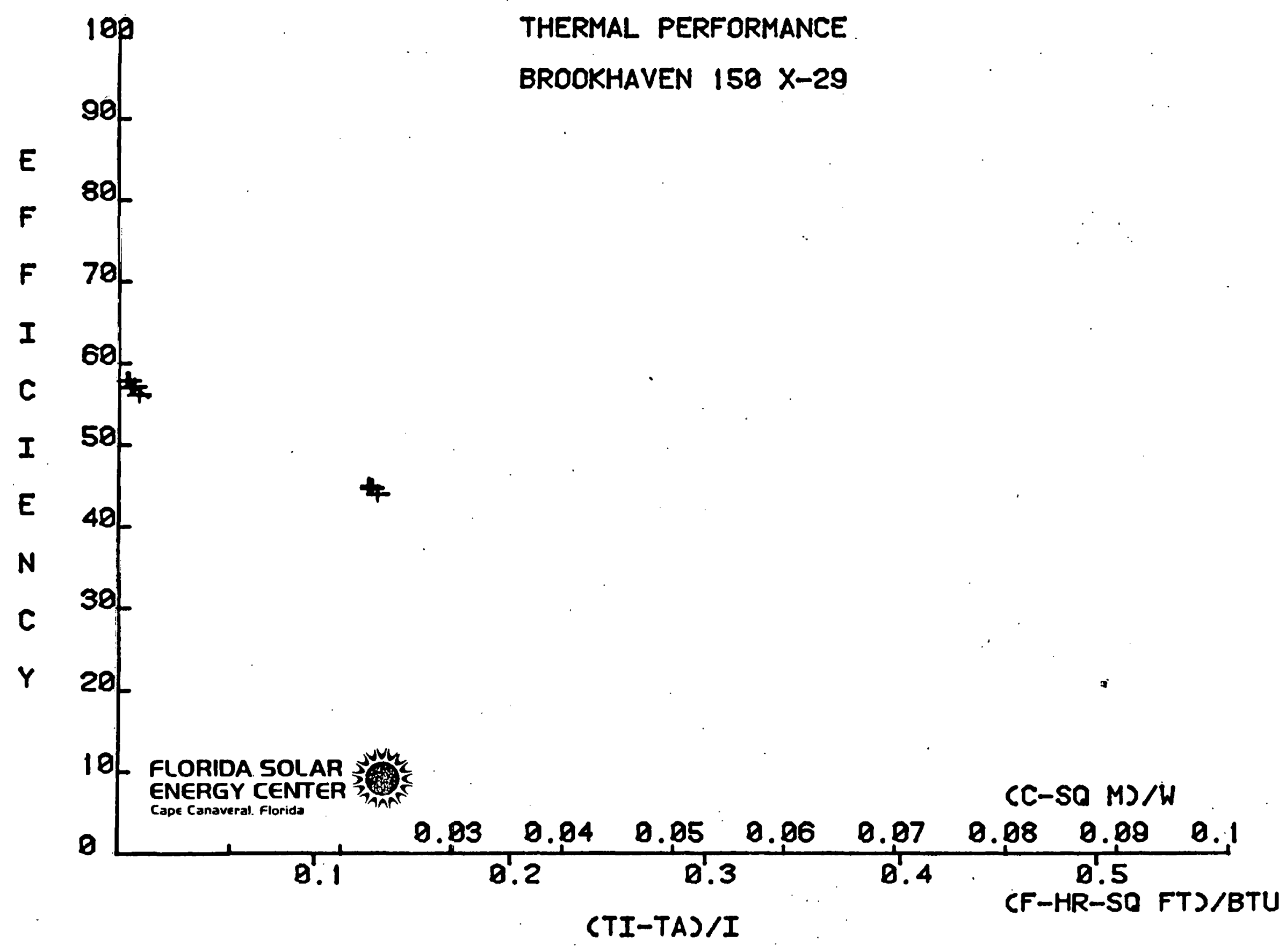




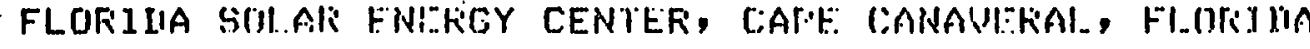
TAINUFACTURER: BFIOKHAUFN $T: \vdots 0$ $\because 0$ I:OO. IIA1 'EST IIATE

i $11 / 9 / 80$ S(II.Ali T]Mr: STAFT FNI! IA TI (i) (i) $1 \mathrm{E}$ MnIr:1.: $x-29$

\section{$211 / 9 / 80$}

$9: 15 \quad 9: 20$

$7.128 .6 \quad 35.4$

27.128.

$35.3 \quad 1.89$

FI.OW

$? 9$

3

4

5

西

\section{6}

$$
\text { , }
$$

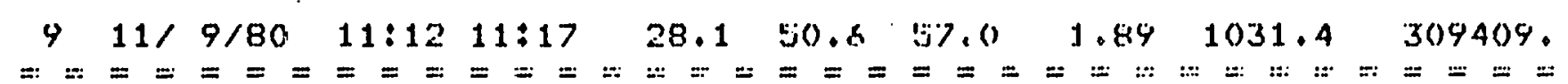

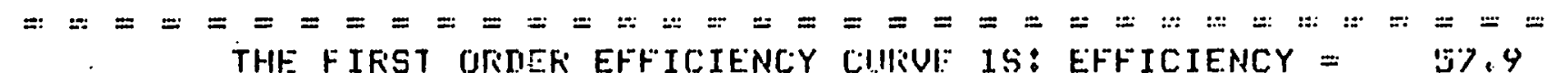
THI: UNITS ON $(T I-T A) / I$ AFIF $(1:-S(N) / N$.

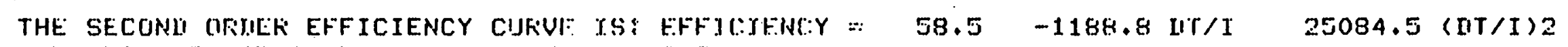
THF. UNITS ON [IT/I AliE $(1 .-5(N) / W$, IIT $K=T I-T A$

\section{$24-A 1: k-81$}

DERSS AliEA $=1.813 \mathrm{SA} \mathrm{N}$

II $T$ EFFIC $(1 I-T A) / T$

(C) $\quad l-M .1 N \quad \% \quad(C-\sin M) / w$

$6.73 \quad 33.7 \quad 56.1 \quad 0.0018$

$6.96 \quad 34.8 \cdot 0.17 .10 .0014$

$7.195 \quad 3 ! 3.7 \quad 57.0 \quad 0.0011$

$\begin{array}{llll}7.68 & 38.4 & 57.8 & 0.0009\end{array}$

$\begin{array}{llll}7.71 & 38.7 \quad 57.8 & 0.00 \% \%\end{array}$

$5.94 \quad 30.0 \quad 43.9 \quad 0.0233$

$\begin{array}{llll}6.02 & \times 0.6 & 44.7 \quad 0.0 \% 8\end{array}$

$6.18 \quad 30.4 \quad 44.8 \quad 0.0225$

$\begin{array}{llll}6.41 & 32.0 & 44.5 & 0.02 \% 18\end{array}$

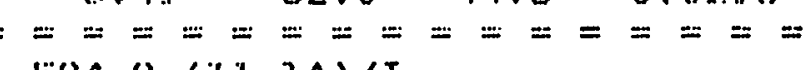
$-1 ; 94.9(11-7 A) / I$

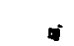




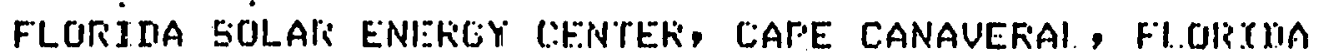

MAINUIFACTUIIEF: BFOOKHAVEN 150

HO15O. LAA

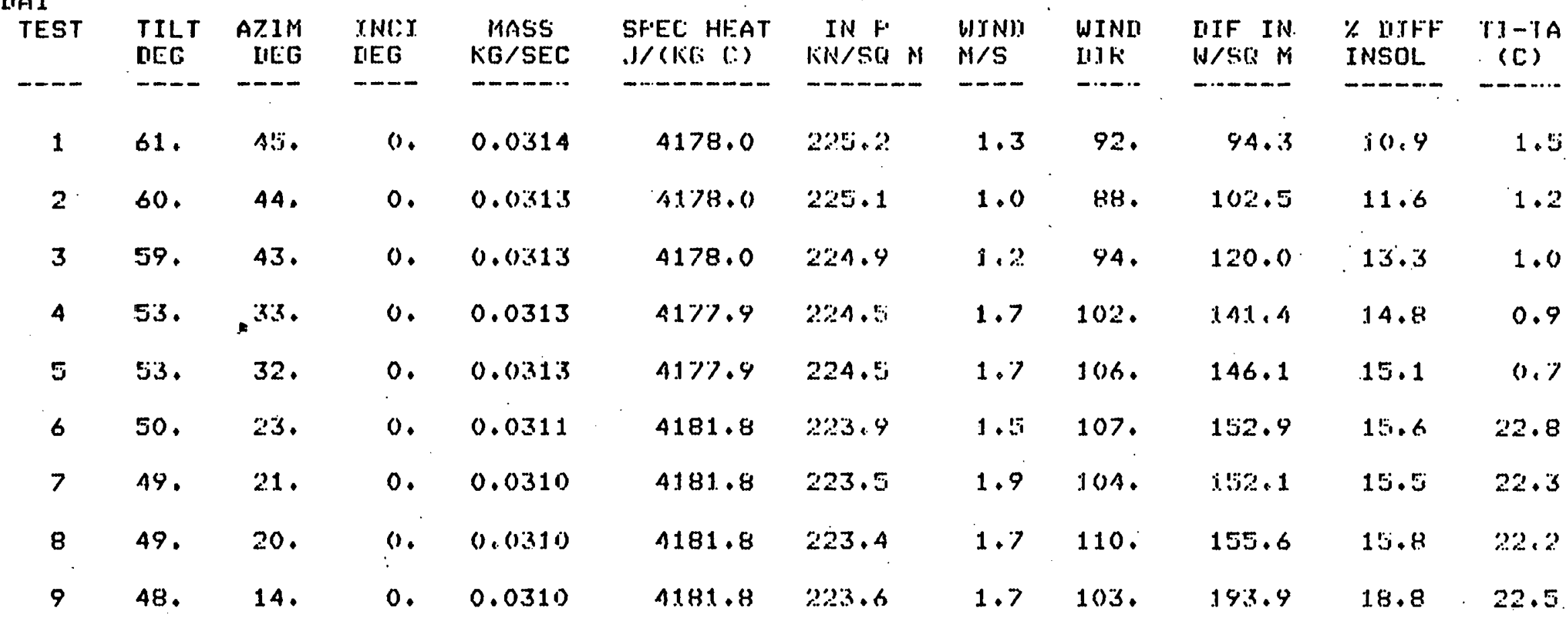




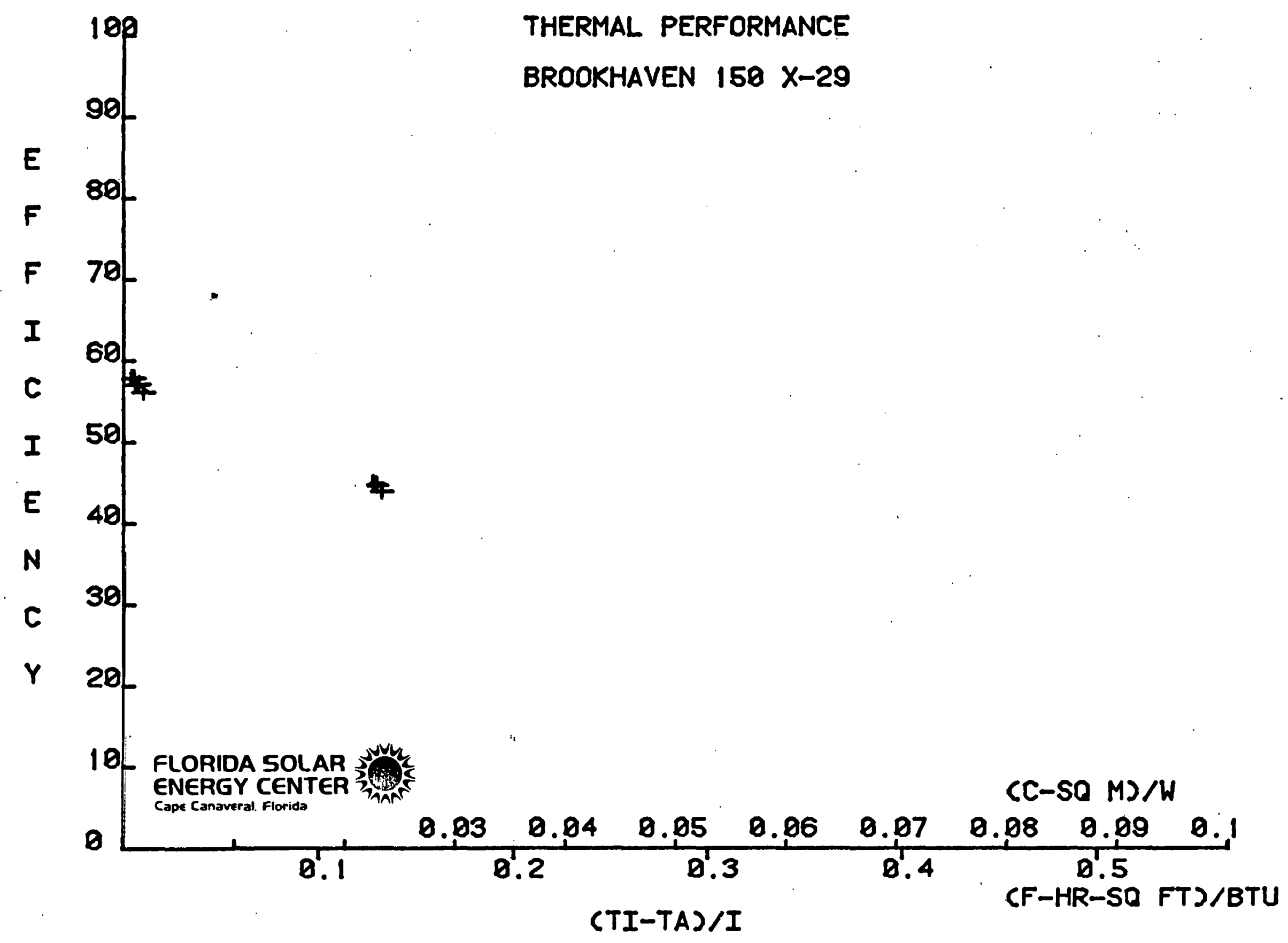


FLURYYIA SUIAAL ENIEKGYY CENTEK, CAFE CANAUTFAL, FI.OFIIIA MRNUIIACTUFEF: BROUKHAULN 150 BO) $\because \because 0 . \mathrm{DA1}$

\begin{tabular}{|c|c|c|c|c|}
\hline TEST & $\begin{array}{l}\text { TILT } \\
\text { NEG }\end{array}$ & $\begin{array}{r}\text { AZIM } \\
\text { IIEG }\end{array}$ & $\begin{array}{l}\text { INCI } \\
\text { IE:G }\end{array}$ & $\begin{array}{c}\text { MASS } \\
\text { I. BNIMIN }\end{array}$ \\
\hline$\therefore$ & $-\cdots$ & $-\cdots-$ & $\cdots \cdots$ & $--\cdots$ \\
\hline
\end{tabular}

Molul:I: $x-294$

$2 A-A_{1} \mid \cdot k-81$

likuss AliEA $=19.51 \mathrm{sQ} \mathrm{F}$

\begin{tabular}{|c|c|c|c|c|c|c|c|c|c|c|c|}
\hline 1 & 61. & 45. & 0. & A. $i ! i$ & 0.998 & $32+7$ & 3.0 & 42. & 29.9 & 10.9 & 2,8 \\
\hline 2 & 60. & 44. & 0. & 4.15 & 0.998 & $32 \cdot 6$ & 2.2 & 88 & 32.5 & 11.6 & $2 \cdot 2$ \\
\hline 3 & 59. & $4: 3$. & 0. & 4.14 & 0.998 & 32.6 & 2.8 & 94. & 38,1 & $13 \cdot 3$ & 1.9 \\
\hline 4 & 53. & 33 & 0. & A. & 0.998 & 32.6 & 3.7 & $1(1) 2$. & 44.9 & 14.8 & 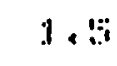 \\
\hline 5 & 53. & 32. & 0. & 4.14 & 0.998 & $32 \cdot 6$ & 3.8 & 106. & 46.4 & $1 r i \cdot 1$ & $1 \cdot 3$ \\
\hline 6 & 50. & 23 & 0 & 4.11 & 0.999 & 32.5 & 3.4 & $i 07$ & 48.5 & 15.6 & 41.0 \\
\hline 7 & 49. & 21. & 0. & 4.10 & 0.999 & 32.4 & 4.2 & 104 & $48 \cdot 3$ & 15.5 & 40,1 \\
\hline 8 & 49. & 20 & 0. & $4 \cdot 10$ & 0.949 & $3 \% 4$ & 3.7 & 110 & 49.4 & 15.8 & 39.9 \\
\hline 9 & 48. & 14. & 0. & 4.11 & 0.999 & 32.4 & 3.8 & $i<13$ & 61.5 & 18.8 & 40.4 \\
\hline
\end{tabular}




\section{APPENDIX B}

PARTIAL DATA

MODEL X-31 
1. COLLECTOR DESCRIPTION

1.1 Collector Identification

Manufacturer and Address:

Brookhaven National Laboratories Solar Technology Group, Bidg. 701 Upton, New York 11973

Collector Nodel Number: $\quad x-31$

\subsection{Collector Construction}

Overall Dimensions:

$\begin{array}{lll}\text { Length: } & 2.337 & \mathrm{~m} \\ \text { Width: } & 0.814 & \mathrm{~m} \\ \text { Depth: } & 0.078 & \mathrm{~m}\end{array}$

Overall Front Area: (length $\times$ width) $=1.903 \mathrm{~m}^{2}$

Height of outlet above inlet when collector tilt is $90^{\circ}: 2.337 \mathrm{~m}$ Glazing:

Material: Dupont Tedlar

Thickness: $0.01 \mathrm{~cm}$

Transmittance*: Not Given

No. of Cover Plates: One

Transparent Frontal Dimensions:
Length:
2.286
m
Width:
0.764
Area:
1.746
m
$\mathrm{m}^{2}$

Absorber:

Material: Two sheets of Tefzel Aluminum laminated together with

Length: $2.286 \mathrm{~m}$ integral fluid passages.

Width: $0.815 \quad \mathrm{~m}$

Area: $1.864 \quad \mathrm{~m}^{2}$

Absorber Coating: $99 \%$ pure nickel foil with selective surface deposits Absorptivity*: top and high temperature silicon adhesive below

Emissivity*: Not Given 
Insulation:

Type: Polyisocyanurate

Dimensions: $3.81 \mathrm{~cm}$. Thr ck

K-Factor ${ }^{\star}: 0.02$ watts $/ \mathrm{m}^{0} \mathrm{C}\left(0.14 \mathrm{Btu} / \mathrm{hr}-\mathrm{ft}^{2}-{ }^{0} \mathrm{~F} / \mathrm{in}\right)$

Heat Transfer Fluid:

Material: Water

Density: $\quad 977.81 \mathrm{~kg} / \mathrm{m}^{3}$ at $70^{\circ} \mathrm{C}$

Specific Heat: $\quad 4189.6 \mathrm{~J} / \mathrm{kg}{ }^{\circ} \mathrm{C}$ at $70^{\circ} \mathrm{C}$

Collector Weight: $7.3 \mathrm{~kg}$

Collector Weight/Area: $\quad 3.84 \mathrm{~kg} / \mathrm{m}^{2}$

Collector Fluid Capacity: $\quad 0.6$ liters

Collector Fluid Capacity/Area: $\quad 0.32$ iiters $/ \mathrm{m}^{2}$

Normal Operating Temperature Range: Not Given ${ }^{\circ} \mathrm{C}$ to ${ }^{\circ} \mathrm{C}$

Manufacturers Recommended Fluid Flow Rate: 1.9 1iter/min ( 0.5 GPM)

Manufacturers Recommended Maximum Operating Pressure:

0. kilopascals ( 0 . psig)

*Information provided by manufacturer; not verified by FSEC. 


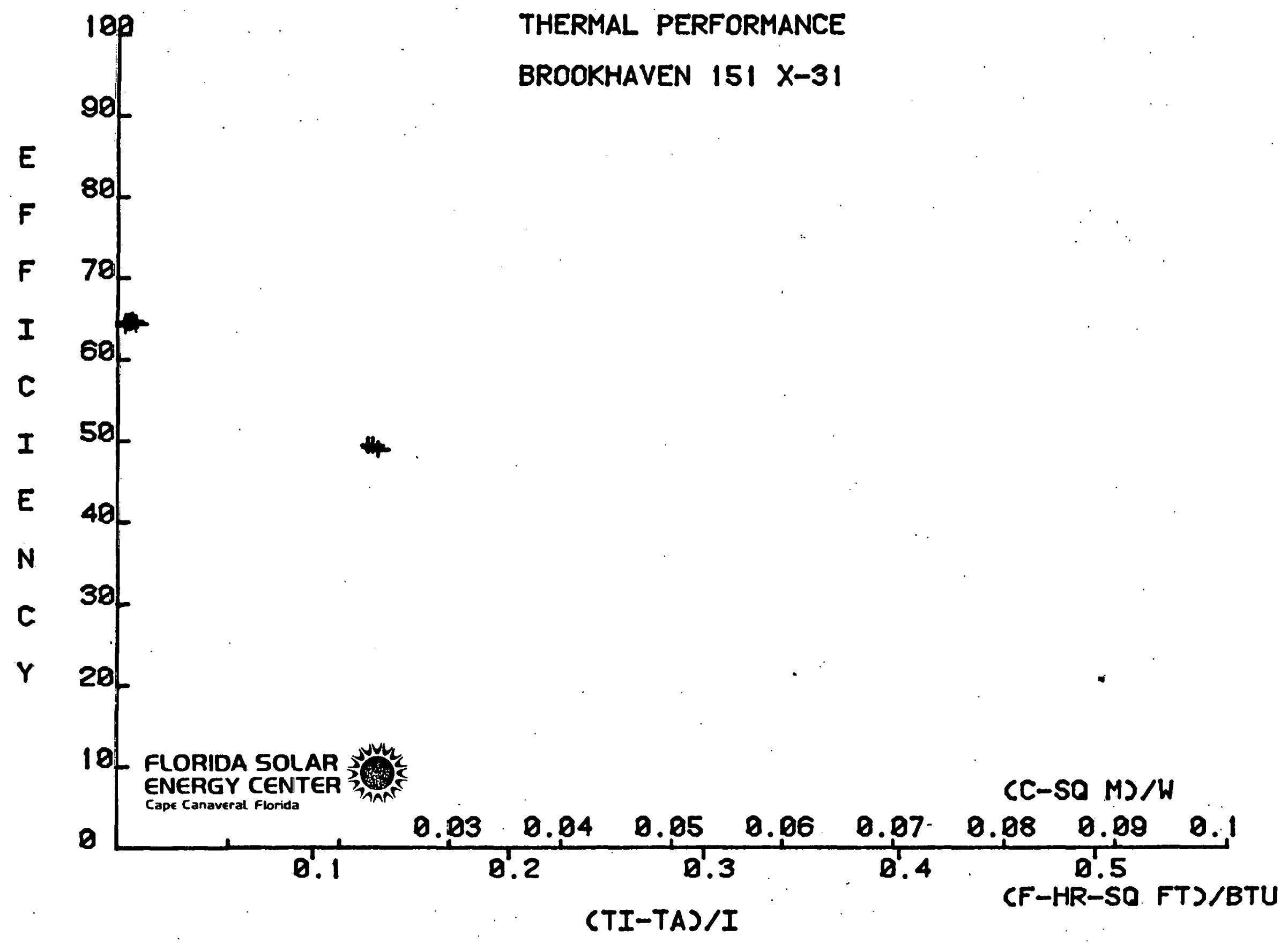




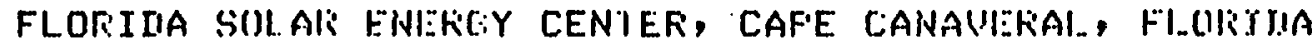

MANIIFACTUFEE: BFOOKHAUI:T! 1 :Gi

Q()i:B. . LIA1

TEST TIATE SULALO TIMI: START FENII

(i) (li) (i:

L/MIN

W/Sl: $M$

i $11 / 9 / 80$

$9: 15 \quad 9: 2: 0$

27.128 .6

36.6

$27.4 \quad 298$.

36.

27.528 .5

37.0

$2 \%, 789.97 \% 3$

1.88

27.828.

28.8150.

$37,4 \quad i .84$

$57.7 \quad 1.88$

28.450 .7

$28.950 .7 \quad 57.8$

$28.150 .6 \quad 5 \%$

$4 \quad 11 / 9 / 80 \quad 11: 1211: 17$

THF FIFST UFIEF: EFFICIENCY CIIISUI $==$

THE: UNITS ON (TI-TA)/I A.IIE $((;-S() ~ M) / W$.

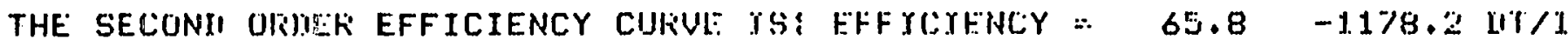

THI: UNITS ON IIT/I AlNE ( :-SQ $M) /(1$, III=TI-TA
I/SR H

$24-A T \cdot K-81$

GROSSS AlitA : 1.903 SR

II. 1 RFFIC $(T I-T A) / I$

(c) $\quad:-N I N \quad$ (r:-SiN $M) / W$

-..... - _ -

$8.015 \quad 46.2 \quad 64.3 \quad 0.0017$

$8.23 \quad 41.2 \quad 64.7 \quad 0.0013$

$8.13 \% \quad 42.2 \quad 64.5 \quad 0.0011$

$8.93 \quad 44.7 \quad 64.6 \quad 0.0008$

$8.99 \quad 45.0 \quad 64.2 \quad 0.0007$

$\begin{array}{llll}7.00 \quad 35.0 & 48.8 & 0.0234\end{array}$

$7.0 \% \quad 35.1 \quad 49.3 \quad 0.0 \% 29$

$7.07 \quad 39.4 \quad 49.3 \quad 0.0225$

$\begin{array}{llll}7.27 & 36.3 & 48.3 & 0.0219\end{array}$

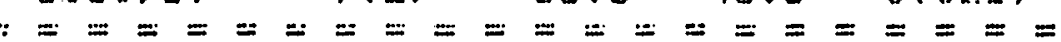
$613.3 \quad-72(1.2(T 1-T A) / I$

$19242.1([\mathrm{IT} / \mathrm{I}) 2$ 


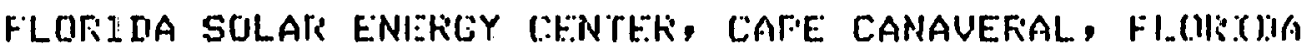
MANUIFACTUKEK: HROOKHAUEN 151 QO i S1 . IIA1

$\begin{array}{llrll}\text { TEST } & \text { TILT } & \text { AZIM } & \text { XNGX } & \text { MASS } \\ & \text { IIEG } & \text { IIEC } & \text { IIEG } & \text { KG/SEC } \\ --- & --- & --- & --- & --\cdots\end{array}$

$3 \quad 59 . \quad 43 . \quad 0.0 .0312$

$453.3 \%$

0.0 .0313

533.32.

0 . (). (1).3.i?

$650 . \quad 23$.

0.0 .0309

$7 \quad 49 . \quad 21$.

0 . 0.0312

849.20.

(1.) 0.00312

948 .

14.

MUIE:L: $x-31$

0 . 0.0310

\begin{tabular}{|c|c|c|c|c|c|c|}
\hline $\begin{array}{l}\text { SFEC HFAT } \\
\text {.1/Rli(: (:) }\end{array}$ & $\begin{array}{l}I N F \\
K N / S C M\end{array}$ & $\begin{array}{l}\text { WNNM } \\
\text { M/S }\end{array}$ & $\begin{array}{l}\text { WINII } \\
\text { IIIK }\end{array}$ & $\begin{array}{l}\operatorname{IIF} I N \\
W / S Q\end{array}$ & $\begin{array}{l}\% \text { MITFF } \\
\text { INSOL }\end{array}$ & $\begin{array}{c}r y-r A \\
(C)\end{array}$ \\
\hline$-\cdots---m$ & $-\infty--\infty$ & $-\cdots-$ & $-\cdots-$ & $---\cdots$ & ----- & $-\cdots--$ \\
\hline $4.17 \% .9$ & 20.2.2 & $1 \cdot 3$ & 92. & 87.7 & $i(1,2$ & 1.5 \\
\hline 4177.9 & $2: 25 \cdot 1$ & 1.0 & 88. & 94.8 & 10.9 & 1.2 \\
\hline 4177.9 & $\therefore ? A_{0}, 9$ & $i .2$ & 94. & 112.8 & $12 \cdot 6$ & 1.0 \\
\hline 4177.4 & $2 \div 4.5$ & 1.7 & 102. & i.36.0 & 14.3 & 0.8 \\
\hline 4177.9 & $224.5 ;$ & 1.7 & 106 & 140.8 & 14.7 & 0.7 \\
\hline 4181.9 & $2: 3,9$ & 1.5 & 107. & 148.1 & 15.2 & 22.8 \\
\hline 1181.9 & 223.5 & 1.4 & $i(14$. & i 48.2 & 15.2 & 32.3 \\
\hline 4181.9 & 223.4 & 1.7 & 110. & 152.0 & 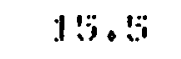 & 22.2 \\
\hline 4.181 .9 & 223.6 & 1.7 & 103. & 189.4 & 18.4 & 22.4 \\
\hline
\end{tabular}

$24-A R \cdot R-81$

GROSS Alit:A :: $:$ i. $9(0 ; 3$ Sil M

$1.7 \quad 103$ 


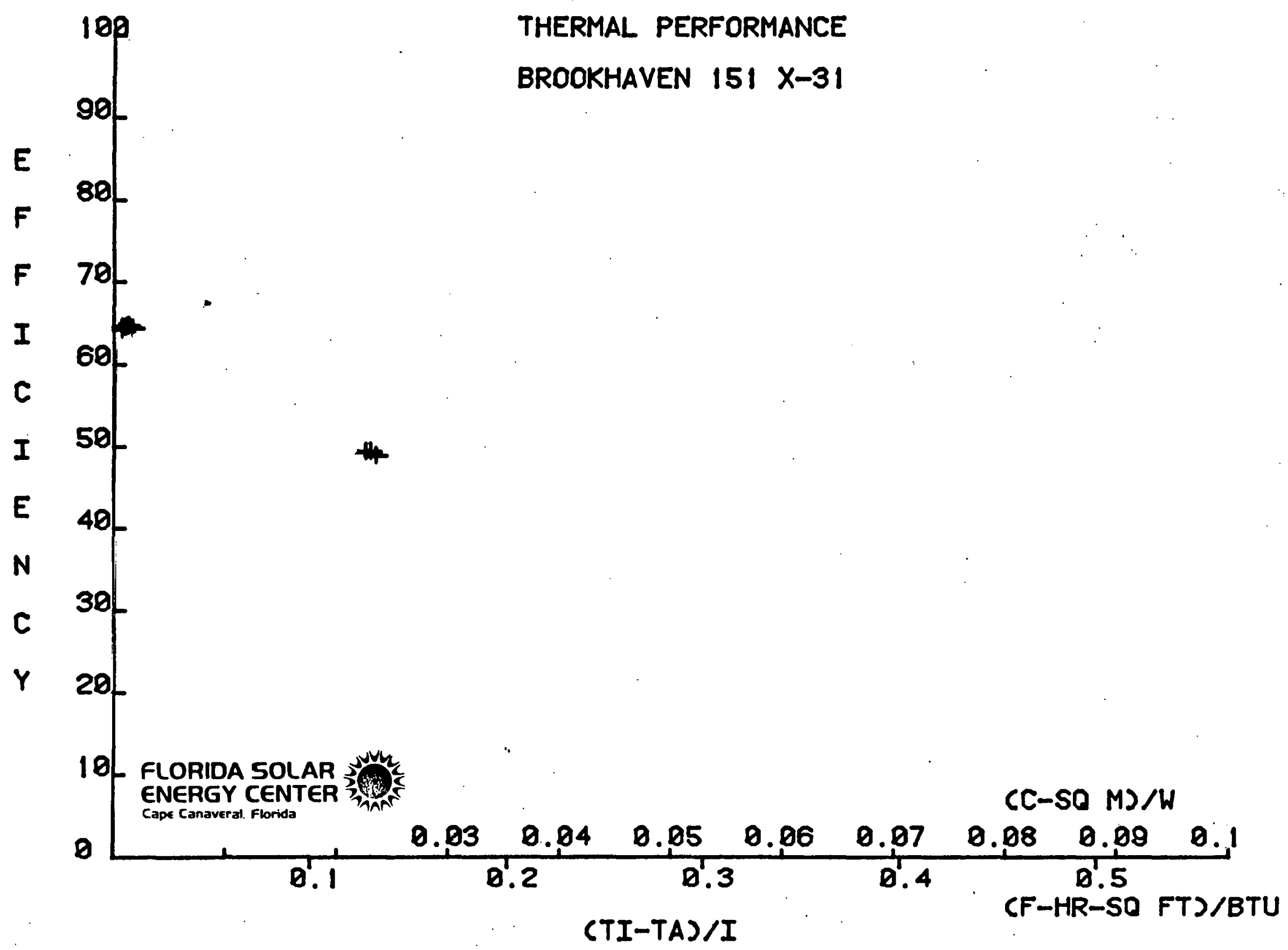




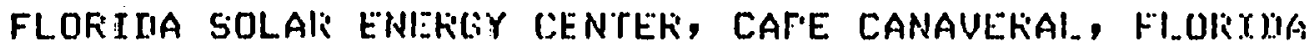
MANUFACTUKEF: BFOOKHAUEN $15 \mathrm{j}$ B() IS1 . LIAI

TEST IIATE

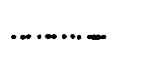

i $11 / 9 / 80$

$211 / 9 / 80$

$311 / 9 / 80$

SOLAR TRMI:

STAFIT ENII

\begin{tabular}{lll} 
(F) & $T I$ & $T E$ \\
(F) & (F) & (F) \\
\hdashline$-\infty$ & - & -
\end{tabular}

NOREL: $\quad x-31$

$411 / 9 / 80$

i) $11 / 9 / 80$ 6 $\%$

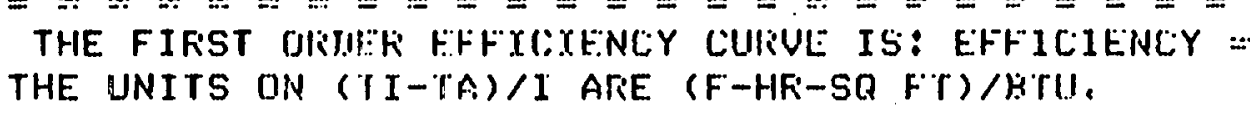

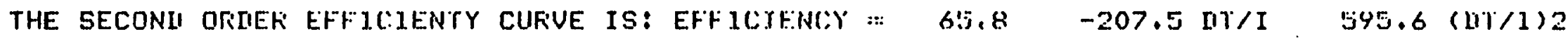

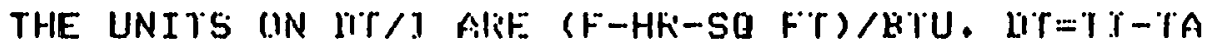


FLIITITIAS SULAR ENEKGY CENTEK, CAFE C:ANAVEKAL, FLOKIIIA

IANUI:ACTUKEF: BFOUKHAVLEN 151

B) $\mathrm{B}: \mathrm{B} 1$. IIA1

\begin{tabular}{|c|c|c|c|c|}
\hline TEST & $\begin{array}{l}\text { TILT } \\
\text { DEG }\end{array}$ & $\begin{array}{r}\text { AZIM } \\
\text { IIEE }\end{array}$ & $\begin{array}{l}\text { INE: } 1 \\
\text { [IE: }\end{array}$ & $\begin{array}{c}\text { MASSS } \\
\text { LBM/MIN }\end{array}$ \\
\hline-- & $\cdots \cdots$ & - & -- & $-m-m-m$ \\
\hline
\end{tabular}

M(1)MU:I.: $x-31$

\section{$21-A_{1} \mid k-81$}

GROSS AREA $=20,48$ SH $F$

\begin{tabular}{|c|c|c|c|c|c|c|c|c|c|c|c|}
\hline 1 & 61. & 45. & 0. & $4 \cdot 14$ & 0.998 & $32+7$ & 3.0 & 92. & 27.8 & $i 0.2$ & $2 ., 7$ \\
\hline 2 & 60. & 14. & 0. & $4 \cdot 14$ & 0.998 & $3 x \cdot 6$ & 2.2 & 88. & 30.1 & 10.9 & 2.1 \\
\hline 3 & 59. & $4 \dot{3}$. & 0 & $4+13$ & 0.998 & 32.6 & 2.8 & 94. & 35.8 & 12.6 & 1.8 \\
\hline 4 & 53. & $=33$ & 0. & $4.1 \mathrm{G}$ & 0.998 & 32.6 & 3.7 & 102. & 43.2 & $i \beta .3$ & 1,5 \\
\hline 5 & 53. & 32. & 0 & 4.13 & $(1.998$ & 32.6 & 3.8 & 106. & 44.7 & 14.7 & 1.2 \\
\hline 6 & 50. & 23. & 0 & 1.05 & 0.999 & 32.5 & 3.4 & $i \leqslant \%$ & 17.0 & 15.2 & 41.0 \\
\hline 7 & 49. & 21. & 0. & $4.1 \overline{3}$ & 0.494 & $3: 4$ & 4.2 & 104 & 47.0 & $i ! 3,2$ & 10.1 \\
\hline 8 & 49. & 20 & 0. & $4 \cdot 13$ & $(1.999$ & 32.4 & 3.7 & 110 & 18.2 & 15.5 & 39.9 \\
\hline 9 & 48. & 14. & 0. & 4.11 & 0.999 & $3 \% \cdot 4$ & 3.8 & $i 013$ & 60.1 & 18.4 & 10.4 \\
\hline
\end{tabular}




\section{APPENDIX C}

PARTIAL DATA

MODEL X-32 


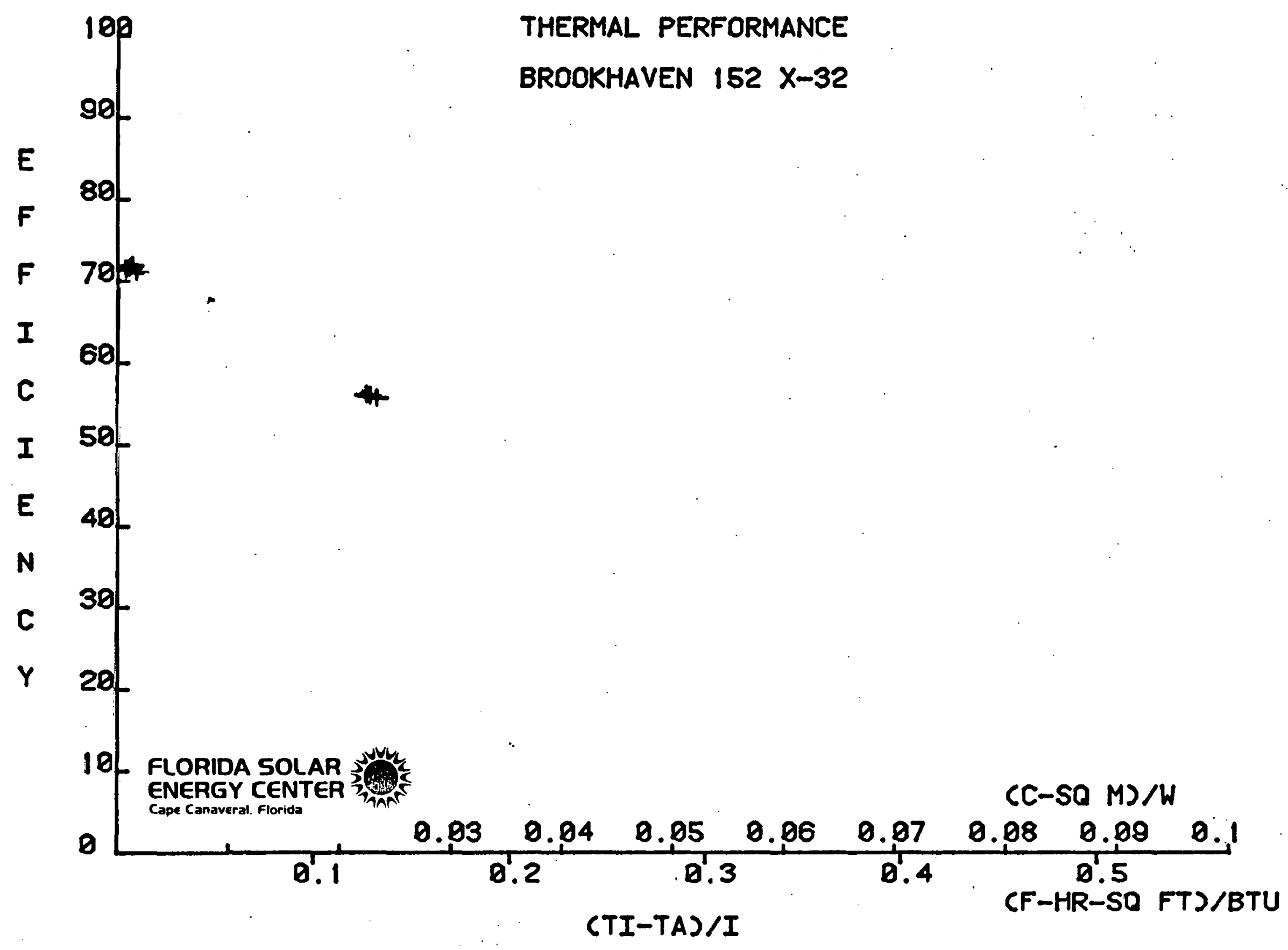




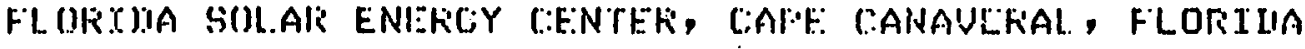

MANUFACTUKEH: HFUUKHAULN 152

(36):I: DA1

TEST NATE

C.....

SOLAR TIME
START ERII

$111 / 9 / 80$

2. $11 / 9 / 80$

$9: 15 \quad 9: 20$

$9: 20 \quad 9: 2: 1$

$4: 27 \quad 9: 32$

$311 / 9 / 80$

$10: 6 * 10: j 1$

$411 / 9 / 80$

$10: 11 \quad 10: 16$

$10: 4 \% 10: 4 \%$

$28.0 \quad 50.5$

28.1

28.

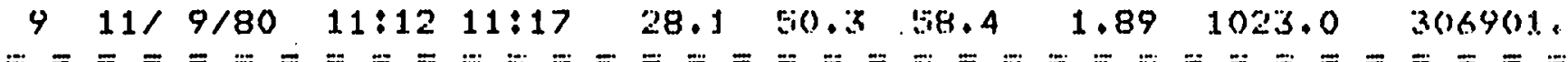

$\begin{array}{ll}\text { TA } & \text { T1 } \\ \text { (C) } & \text { (C) } \\ -\cdots & -\cdots\end{array}$

M(1)MEI.: $x-32$

$8 \quad 11 / 9 / 80 \quad 10: 5511: 0$

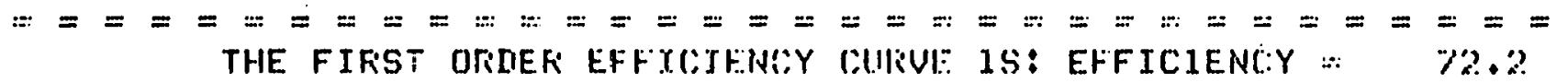
THE FIFST ORIDER EFFXCIFINGY CUIIVI: 1S: EFFICIENC:Y : THE: UNITS DN $(1-1-T A) / 1$ AlitE $(C:-S Q M) / W$.

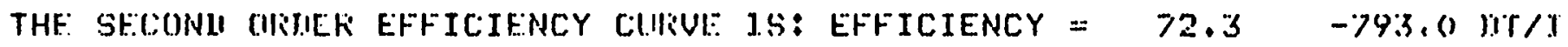

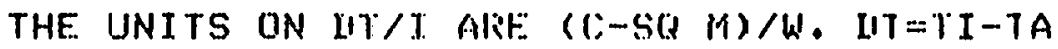

$24-A l: k i-81$.

CIROSS AREA $=1.816 \mathrm{SIR} \mathrm{K}$

DEL T EFFIC. $(r x-T(A) /]$ (:-MIN $\% \quad(C-S(1) M) / W$ (C) -

$8.13 \quad 12.2 \quad 71.0 \quad 0.001 \%$

$8.6 \% \quad 43.3 . \% 1.8 \quad 0.0013$

$8.90 \quad 44.5 \quad 71.5 \quad 0.0011$

$\begin{array}{llll}9.45 & 17.2 & 71.3 & 0.0008\end{array}$

$4.6 \% \quad 47.6 \quad 71.4 \quad 0.000 \%$

$7.60 \quad 38.0 \quad 513.7 \quad 0.0232$

$\begin{array}{llll}7.65 & 38.2 & 515.9 & 0.0 \% 28\end{array}$

$\begin{array}{llll}7.72 & 38.6 & 56.1 & 0.0223\end{array}$

$\begin{array}{llll}8.03 & 40.1 \quad 56.2 & 0.0 \% 17\end{array}$

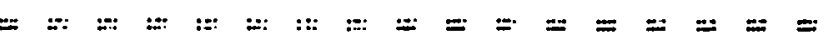
$.721 .9(T I-T A) / T$

$72 \cdot 3$

$3119.3(117 / 1) 2$ 


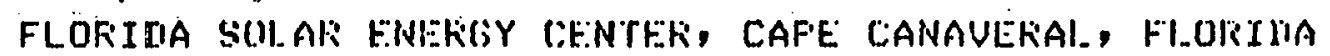

MANUIFACTUKEK: BFOOKHAUEN $15 \%$

BOUI52. IIA1

\begin{tabular}{|c|c|c|c|c|}
\hline TEST & $\begin{array}{l}\text { TILT } \\
\text { DI: }: \text { T }\end{array}$ & $\begin{array}{l}\text { AZIM } \\
\text { IIE:G }\end{array}$ & $\begin{array}{l}\text { Yine: } \\
\text { hil:c: }\end{array}$ & $\begin{array}{l}\text { MASS } \\
K G / S E C\end{array}$ \\
\hline-1 & $-\infty-$ & $-\cdots$ & --- & $\therefore$ \\
\hline
\end{tabular}

MOLIEL: $X-32$

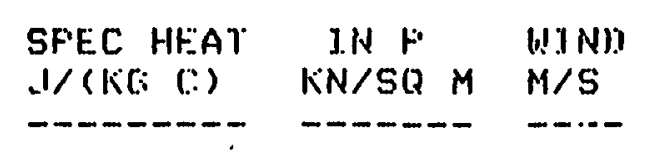

\section{$24-A C \cdot R-B 1$}

CKOSS Alit:A :: 1.816

\begin{tabular}{|c|c|c|c|c|c|c|c|c|c|c|}
\hline 61. & 45 & 0. & 0.0313 & 4177.4 & 285.2 & $1 \cdot 3$ & 92. & $8 A \cdot 6$ & 9.9 & 1.5 \\
\hline 60. & 14. & 0 & 0.0313 & 1177.4 & $225 \cdot 1$ & 1.0 & 88. & 91.8 & 10.5 & 1.2 \\
\hline 59. & 43 & (). & $(1.0313$ & 4177.9 & 224.9 & 1.2 & 94. & 110.1 & $1.2+3$ & 1.0 \\
\hline 53 & 33 & 0 & 0.0312 & $4.17 \% .4$ & 224.6 & 1.7 & $10 \%$ & $134 \cdot 0$ & $14 \cdot 1$ & 0.8 \\
\hline 53. & 32. & 0 & 0.0312 & 4.177 .9 & 224.5 & 1.7 & $i 06$. & 138.8 & 14.3 & 0.7 \\
\hline 50. & 23. & (1. & $0.030 \%$ & 4181.9 & $2,23,4$ & 1.5 & 107 & 145.4 & 193.0 & 5'.2. \\
\hline 19. & 21. & 0 & 0.0309 & A.iBl. 9 & ن & 1.9 & 104. & 144.8 & 14.9 & 200 \\
\hline 49. & 20 & 0. & 0.013019 & 4181.9 & 223.1 & 1.7 & 110. & 148.6 & $13 \cdot 2$ & 2.1 .9 \\
\hline 48. & 14. & 0. & 0.0311 & 4182.0 & 283.6 & 1.7 & 103. & 18: & 18.1 & 2.2 \\
\hline
\end{tabular}




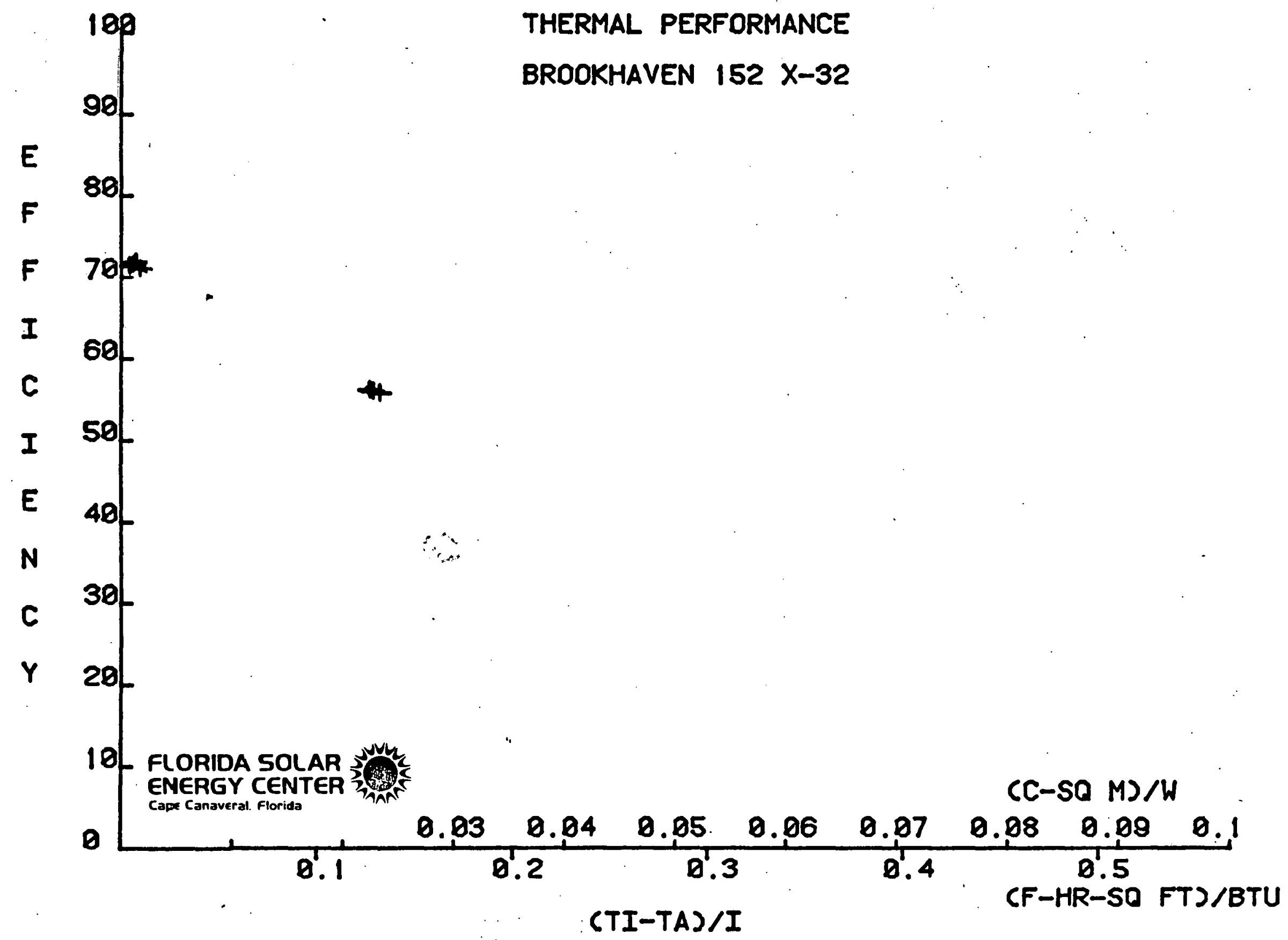




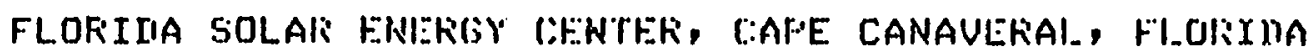
MANLIFACTUKER: BROOKHAUEN 152 (3)152, IIA1

TEST MATE

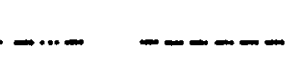

$111 / 9 / 80$

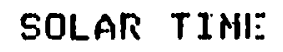

START ENII

$\begin{array}{lll}\text { TA } & \text { TI } & \text { TE. } \\ \text { (F) } & \text { (F) } & \text { (F) } \\ -\cdots & -\cdots & ---\end{array}$

MOIIEL: $X-32$

$? 11 / 9 / 80$

$\begin{array}{llllll}4: 15 & 9: 20 & 80.7 & 83.4 & 98.6 & 0.494\end{array}$

$311 / 9 / 80$

$\begin{array}{llllll}9: 20 & 9: 25 & 81.2 & 83.3 & 98.4 & 0.494\end{array}$

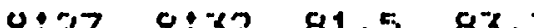

49.30 .948

$9: 27 \quad 9: 32$

$411 / 9 / 80$

$10: 6,10: 1$

8.1.

B.

$10: 11 \quad 10: 16 \quad 82$.

$83.3 \quad 100.3 \quad 0.497$

497

ij $11 / 9 / 80$

b $11 / 9 / 80$

$10: 42,10: 47$

8:3 3122.8

$500.3 \quad 0.498$

$3(1) 1.8$

303.8

$136.5 \quad 0.197 \quad 361 \% .9$

$4 \quad 11 / 9 / 80 \quad 11: 12 \quad 11: 1 \% \quad 82.6 \quad 122.6 \quad 13 \% .0(1 .: 3(1) \quad 324.5$

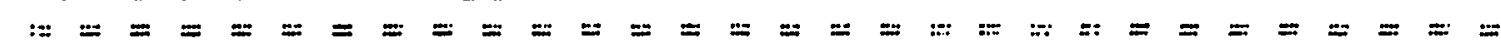

THF. FIRST URMIER EFFIC:IENCY CUHUVI: IS: EFFICIENC:Y, THE UNITS UIN (1T-TA)/I ARE (F-HK-S() FT)/HIU.

THE SECONII CIRNER FHFICIENTY CURUL IS: H.FFIG:XENLY

THE UNITS UN IIT/I. ARE (F-HR-S(A FT)/ATU. IIT=TI-TA 
FLOHILA SULAR ENIEKTSY CEENTHE, TEATE CANAULFAI., FLURMMA

MANUI:ACTURER: HFUDKHAUEN 152

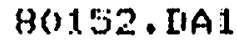

\begin{tabular}{|c|c|c|c|c|}
\hline$T \equiv S T$ & $\begin{array}{l}\text { TILT } \\
\text { LUEG }\end{array}$ & $\begin{array}{l}\text { AZ1M } \\
\text { LI:CG }\end{array}$ & $\begin{array}{l}\text { rNC:Y } \\
\text { PEG }\end{array}$ & $\begin{array}{c}\text { MASS } \\
\text { LEM/NIN }\end{array}$ \\
\hline & $\ldots$ & $---m$ & $-\cdots$ & $-\cdots$ \\
\hline
\end{tabular}

Munt:I.: $x-32$

3

4$$
4
$$

5 53. 32. 0.01 .1

6 50. 23. 0.4 .0

$749 . \quad 21.40 .08$

849.20 .20 .049

948.44 .4 .11
$0.498 \quad 3 \% .7$

$0.948 \quad 3 \% .6$

$0.998 \quad 32.6$

$0.998 \quad 3 \because+6$

$0.498 \quad 32.6$

$0.999 \quad 3 \because . \because$

0.494

0.949

0.999
$3 x+4$

324

32,4
$24^{\circ}-A R \cdot F-81$

CROSS AREA $=19,195$ SN F

$\begin{array}{lcccc}\text { WINM } & \begin{array}{l}\text { WINL } \\ \text { MIYH }\end{array} & \begin{array}{l}\text { IIF IN } \\ \text { K/H.1.72 }\end{array} & \begin{array}{c}\text { MIFF } \\ \text { INSOL }\end{array} & \begin{array}{c}\text { IX-TA } \\ \text { (F) }\end{array} \\ 3.0 & 92 . & 26.8 & 9.9 & 2.6 \\ 2.2 & 88 . & 29.1 & 10.5 & 2.1 \\ 2.8 & 94 . & 34.9 & 12.3 & 1.8 \\ 3.7 & 102 . & 42.9 & 14.1 & 1.4 \\ 3.8 & 106 . & 14.0 & 14.5 & 1.2 \\ 3.4 & 10 \% & 46.1 & 15.0 & 40.5 \\ 4.2 & 104 . & 45.9 & 14.9 & 39.6 \\ 3.7 & 110 . & 47.1 & 15.2 & 3 \% .4 \\ 3.8 & 103 . & 58.8 & 18.1 & 43.0\end{array}$




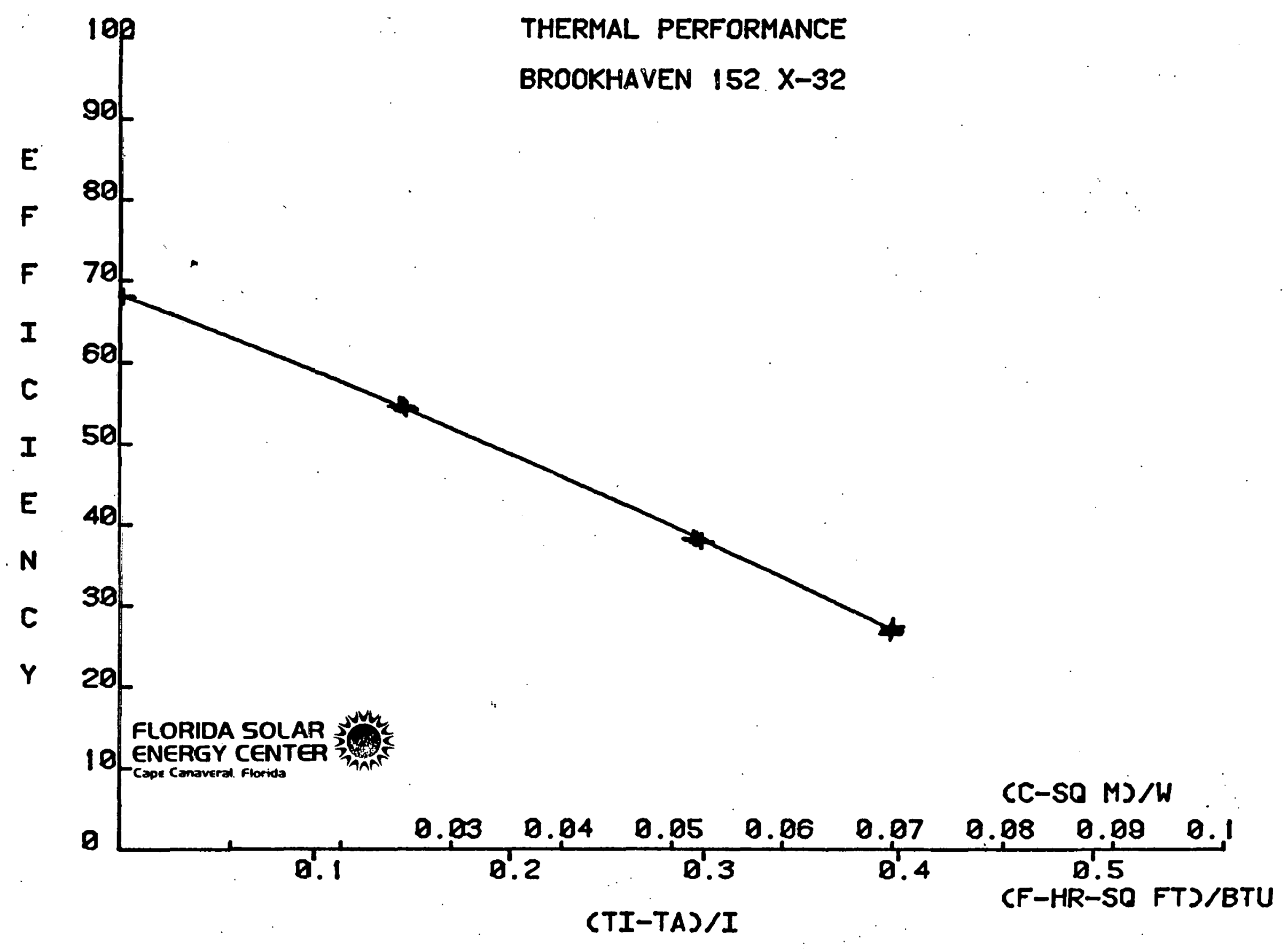





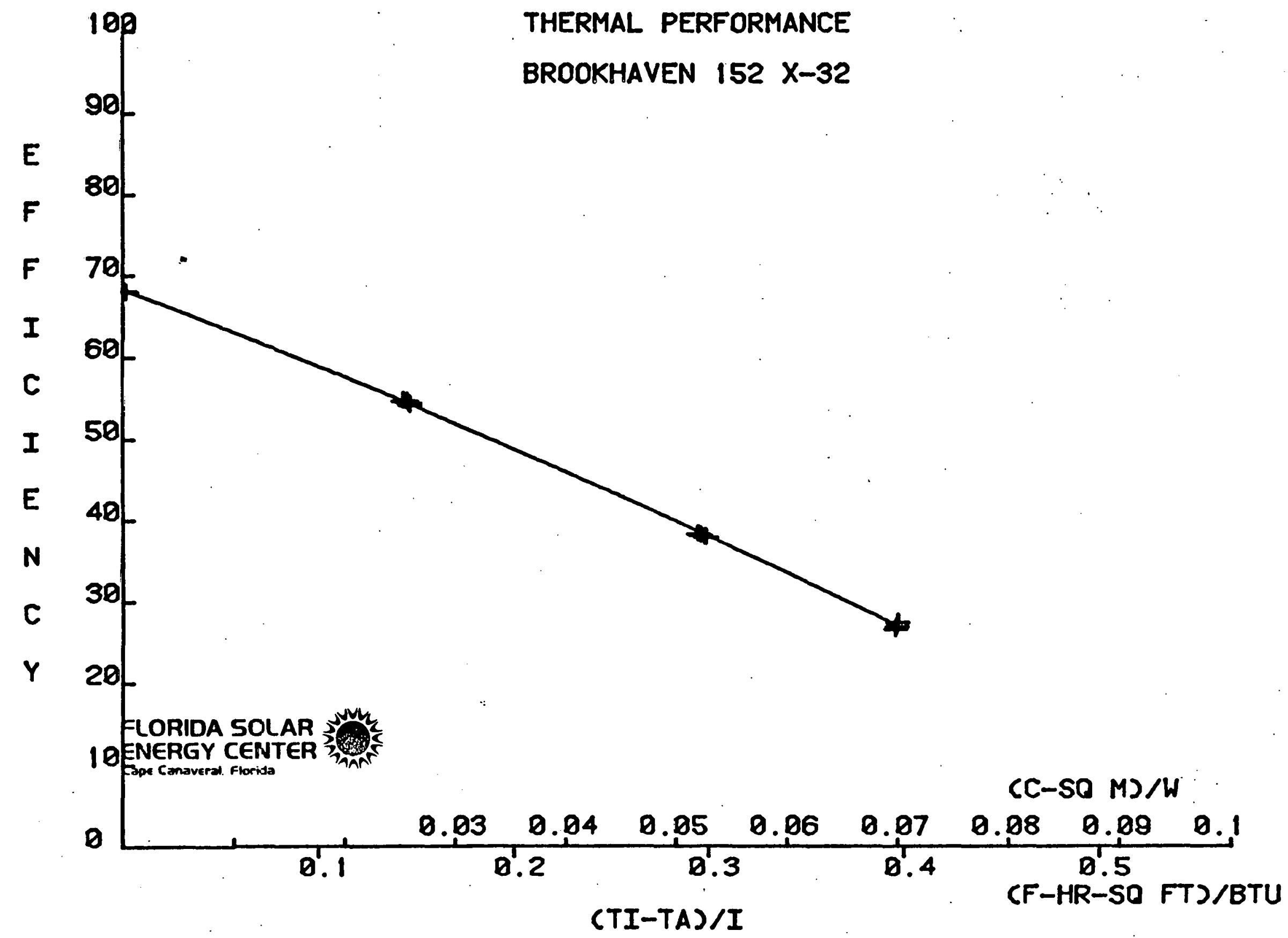




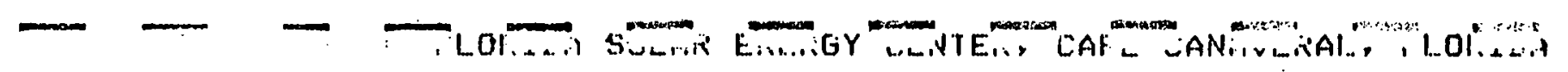
MANUFACTUFEF: RROOKHAUEN 15:

\begin{tabular}{|c|c|c|c|c|c|c|c|c|c|c|c|c|c|}
\hline IEST & IIATE & $\begin{array}{l}\text { SOLAK } \\
\text { STAK'1 }\end{array}$ & $\begin{array}{l}\text { TIME } \\
\text { ENII }\end{array}$ & $\begin{array}{l}\text { TA } \\
\text { (F) }\end{array}$ & $\begin{array}{l}T I \\
(F)\end{array}$ & $\begin{array}{l}T E \\
(F)\end{array}$ & $\begin{array}{l}\text { FLOW } \\
\text { GF'M }\end{array}$ & $\begin{array}{l}\text { AUE IN } \\
\text { B/H -FT2 }\end{array}$ & $\begin{array}{l}\text { TCITAL. IN } \\
\text { ETU/SR FT }\end{array}$ & $\begin{array}{c}(T E-T I) \\
(F)\end{array}$ & $\begin{array}{l}\text { IEL T } \\
F-M I N\end{array}$ & $\begin{array}{c}\text { EFF } \\
\%\end{array}$ & $\begin{array}{l}\text { (TI-TA } / I \\
\text { (F-H-FT2 }\end{array}$ \\
\hline$\cdots-\cdots-$ & $-\cdots-\infty$ & $-\infty-\infty$ & $----\infty$ & $-\infty-\infty$ & --- & --- & --- & $-\cdots-\cdots$ & $-----\cdots$ & $-\infty-\infty$ & ---- & -- & ------- \\
\hline 1.3 & $11 / 25 / 80$ & $9: 47$ & $9: 52$ & 70.6 & 161.5 & 170.8 & 0.501 & 307.4 & 25.6 & 9.36 & 46.8 & 38.2 & 0.296 \\
\hline 14 & $11 / 25 / 80$ & $9: 52$ & $9: 57$ & 71.0 & 161.2 & 170.6 & 0.500 & 306.2 & 25.5 & 9.38 & 46.9 & 38.3 & 0.295 \\
\hline 15 & $11 / 25 / 80$ & $9: 57$ & $10: 2$ & 71.4 & 161.2 & 170.4 & 0.500 & $301 \cdot 6$ & 25.1 & 9.13 & $45 \cdot 7$ & 37.9 & 0.298 \\
\hline 16 & $11 / 25 / 80$ & $10: 2$ & $10: 7$ & 71.4 & 161.2 & 170.3 & 0.500 & 300.8 & 25.1 & 9.12 & 45.6 & 37.9 & 0.299 \\
\hline 21 & $11 / 25 / 80$ & $10: 53$ & $10: 58$ & 72.7 & 196.1 & 202.4 & 0.500 & 311.7 & 26.0 & 6.78 & 33.9 & 27.0 & 0.396 \\
\hline 22 & $11 / 25 / 80$ & $10: 58$ & $11: 3$ & 73.0 & 196.3 & 203.2 & 0.501 & 310.8 & 25.9 & 6.88 & 34.4 & 27.5 & 0.397 \\
\hline 23 & $11 / 25 / 80$ & $11: 3$ & $11: 8$ & 72.7 & 196.1 & 202.8 & 0.501 & 311.9 & 26.0 & 6.69 & 33.5 & 26.7 & 0.396 \\
\hline ?5 & $11 / 25 / 80$ & $11: 40$ & $11: 45$ & 73.1 & 119.1 & 132.7 & 0.499 & $31: 3$ & $26 \cdot 3$ & 13.58 & 67.9 & 54.3 & 0.146 \\
\hline 266 & $11 / 25 / 80$ & $11: 45$ & $11: 50$ & 73.1 & 119.2 & 132.8 & 0.499 & 315.6 & 26.3 & 13.58 & 67.9 & 54.2 & 0.146 \\
\hline $2 B$ & $11 / 25 / 80$ & $11: 55$ & $12: 0$ & 73.1 & 118.7 & 132.4 & 0.498 & 315.4 & $2: 6 \cdot 3$ & 13.70 & 68.5 & 54.6 & 0.145 \\
\hline 29 & $11 / 25 / 80$ & $12: 0$ & $12: 5$ & 73.2 & $118 \cdot 4$ & 132.1 & 0.499 & $316 \cdot 3$ & 26.4 & $13 \cdot 71$ & 68.5 & 54.7 & 0.143 \\
\hline 30 & $11 / 25 / 80$ & $12: 30$ & $12: 35$ & 73.5 & 74.1 & 90.9 & 0.500 & 315.5 & 26.3 & 16.80 & 84.0 & $6 \% .9$ & 0.002 \\
\hline 32 & $11 / 25 / 80$ & $12: 40$ & $12: 45$ & 73.6 & 74.0 & 90.9 & 0.500 & 314.8 & 26.2 & 16.84 & 84.2 & 68.1 & 0.001 \\
\hline $\begin{array}{l}3.3 \\
==\end{array}$ & $\begin{array}{c}11 / 25 / 80 \\
== \\
\text { THE } \\
\text { THE U }\end{array}$ & $\begin{array}{l}12: 45 \\
=== \\
\text { FIFST } \\
\text { INITS ON }\end{array}$ & $\begin{array}{l}12: 50 \\
=== \\
\text { JREER } \\
\text { RTI- }\end{array}$ & $\begin{array}{l}\quad 73.6 \\
=== \\
\text { EFFIC:IE } \\
\text { (A) } / I\end{array}$ & $\begin{array}{l}74.0 \\
=== \\
\text { ENCY CU } \\
\text { AFE (F- }\end{array}$ & $\begin{array}{l}\quad 90.8 \\
==2= \\
\text { FUE IS: } \\
H K-S G \mathrm{FI}\end{array}$ & $\begin{array}{l}0.499 \\
=== \\
\text { EFFICI } \\
\text { I)/BIU. }\end{array}$ & $\begin{array}{l}31.4 \cdot 4 \\
=== \\
\text { IENCY }=\end{array}$ & $=\frac{26.2}{=}==$ & $\begin{array}{l}16.80 \\
==== \\
-104.1111\end{array}$ & $\begin{aligned} & 84.0 \\
= & == \\
-(A) / 1 & =\end{aligned}$ & $\begin{array}{l}68.0 \\
===\end{array}$ & $=\begin{array}{r}0.001 \\
===\end{array}$ \\
\hline & $\begin{array}{l}\text { THE S } \\
\text { THE U }\end{array}$ & $\begin{array}{l}\text { ECONII } \\
\text { INITS ON }\end{array}$ & $\begin{array}{l}\text { DFIIIEK } \\
\text { I IIT/I }\end{array}$ & $\begin{array}{l}\text { EFFIC:L } \\
A F E \text { C }\end{array}$ & $\begin{array}{l}\text { ENTY CU } \\
F-H F-S Q\end{array}$ & $\begin{array}{l}\text { FUE IS: } \\
F I \text { IIIL }\end{array}$ & $\begin{array}{l}\text { EFFICI } \\
\text { J. III }=T\end{array}$ & $\begin{array}{l}\text { IENCY = } \\
I I-T A\end{array}$ & 68.2 & $91.3 \mathrm{IIT} / \mathrm{I}$ & - & 311 & (I) 2 \\
\hline
\end{tabular}

MOIIEL: $\quad x-32$ 


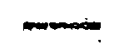

MOIIEL: $\quad x-32$

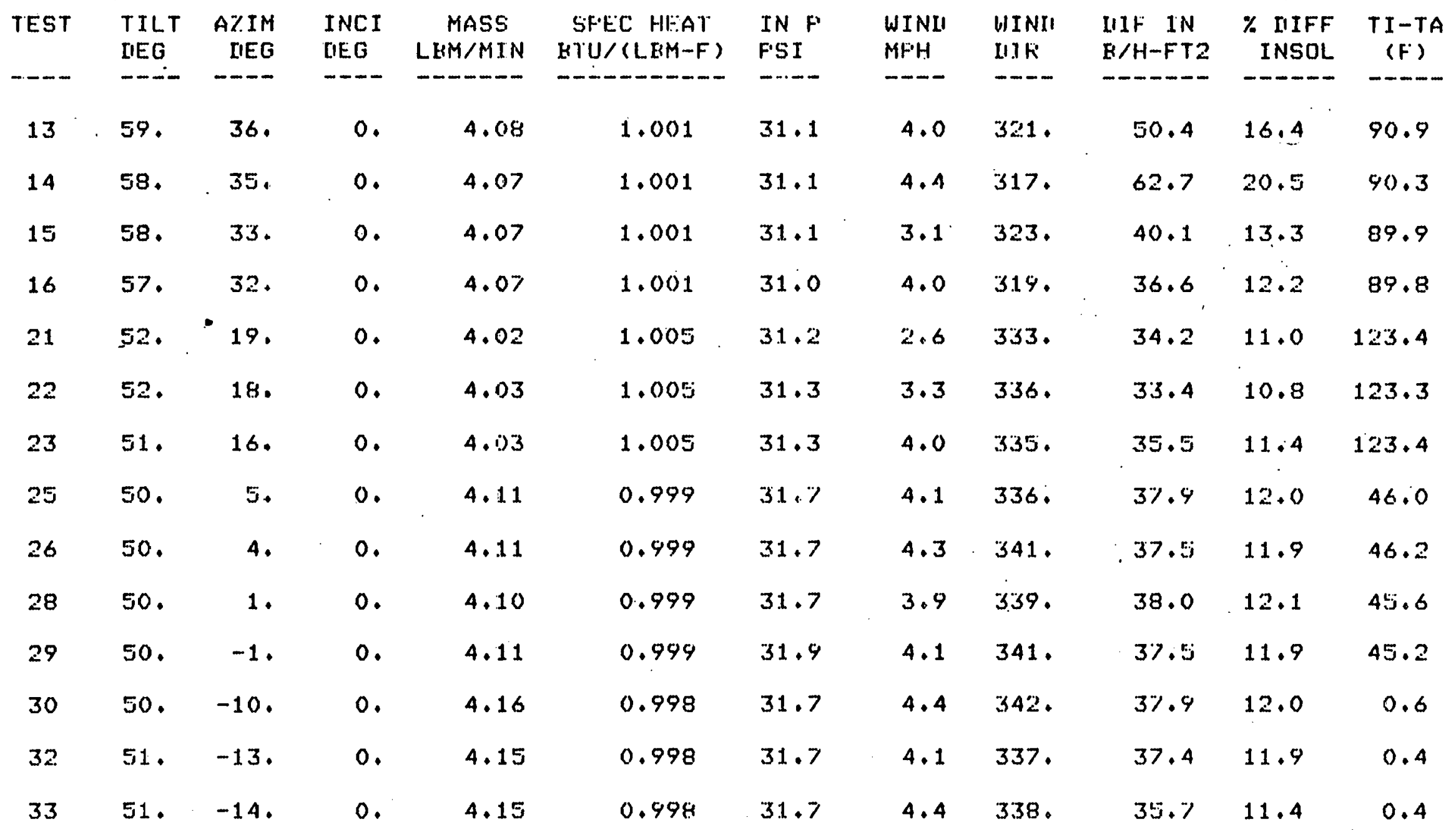




\section{FLORIDA SOLAR ENERGY CENTER}

300 State Road 40I. Cape Canaveral. Florida 32920. Telephone: (305) 783-0300

State University System of Florida

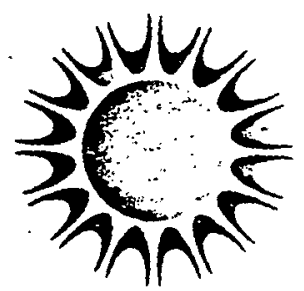

June 9, 1981

William G. Wilhelm

Brookhaven National Iaboratory

Building 701

Upton, New York 11973

Dear Bill:

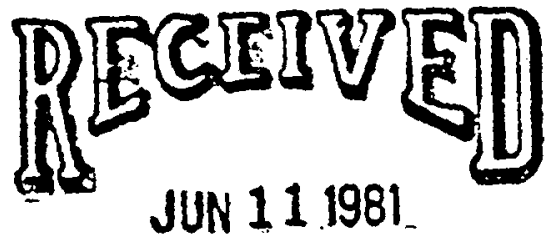

SOUTHERA SOLAR ENERGY

CENTER

Enclosed is a revision sheet to the test report on your Model $x-29, x-31$ and $x-32$ solar collectors. The specific change is a more detailed description of the condition of the Model $\mathrm{X}-32$ after exposure.

The initial report stated that "the absorber buckled." A close reexamination showed that what was described as buckling were a number of creases caused by loss of adhesion between the absorber coating laminate and the absorber itself along the fusion lines of the absorber. The adhesive did not degrade above the fluid flow path but only above the fusion lines.

I understand that all of the degradations that appeared after exposure were due to the prototype nature of the collector. We were told when the collectors were delivered that problems were expected with the adhesive and that the adhesive material was being changed. Breakdown of the foil tape holding the collector together was not totally unexpected either. I assume the foil tape was only a temporary measure. Certainly, other methods of fastening the enclosure are available.

Overall, I was impressed with the results. Perfomance of the collector compares favorably. with othor collectors of its type. A trial energy rating, in accordance with the Floxida rating method, shows that the collector produces 14,800 BTU per day at the intermediate temperature (122\% F). Energy rating per gross area is 757 BIU per square foot. collectors with higher ratings generally use glass as a cover plate. The only plastic glazed collectors with higher intermediate ratings used two covers or used a black chrome selective surface on copper sheet and tube. Enclosed is a copy of the March 1981 Thermal Performance Ratings of the FSEC approved solar collectors. You may wish to make your own comparison.

Based on the thermal performance results, I believe further develogment is justified. If the collector can be produced for relatively low cost, its potential is great:

If I can be of further assistance or if you have any questions, please contact me.

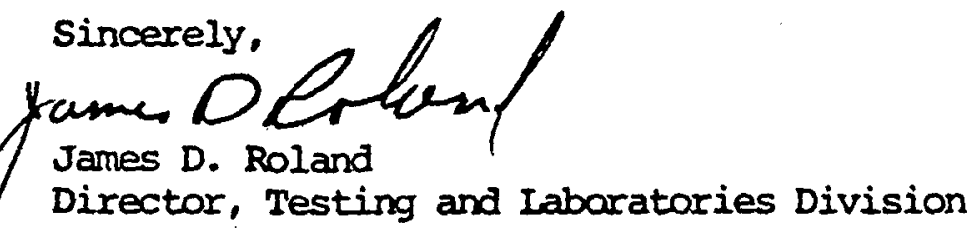




\section{Thin-Film Plastic Solar Collector \\ Technical Review \\ January 13, 1981 \\ Brookhaven National Laboratory}

$\begin{array}{ll} & \text { Agenda } \\ 8: 30-9: 00 \text { AM } & \text { Coffee and Danish } \\ 9: 00-9: 10 \text { AM } & \text { Opening Remarks, T.E. O'Hare } \\ 9: 10-10: 00 \text { AM } & \text { Thin-Film Plastic Collector Update, } \\ \text { W.G. Wilhelm } & \text { Coffee } \\ 10: 00-10: 15 \text { AM } & \text { Questions } \\ 10: 15-11: 00 \text { AM } & \text { Field Visit - Collector Fabrication } \\ 11: 00-12: 00 \text { AM } & \text { Test Facility } \\ 12: 00-3: 00 \text { PM } & \text { Lunch } \\ 1: 00-3: 30 \text { FM } & \text { Deliberation of Comittee } \\ 3: 30-3: 45 \text { FM } & \text { Coffee } \\ 3: 45-4: 45 \text { PM } & \text { Discussion of Comittee Findings }\end{array}$




\section{LIST OF ATTENDEES}

Thin-Film Plastic Solar Collector

Technical Review

January 13, 1981

Mr. Kirk Collier

Solar Energy Research Institute

1536 Cole Boulevard

Golden, $\mathrm{CO} 80401$

(FTS) $327-1761$

Mr. G. W. Knowles

Grumman Energy Systems, Inc.

10 Orville Drive

Bohemia, NY 11716

(516) 244-2786

Mr. Bud Nickelson

Polymer Products Department

E. I. DuPont DeNemours \& Co.

Wilmington, DE 19898

(302) 744-8695

Dr. Louis A. Ross

General Manager

Polymer and Plastics Technology

The Continental Group,. Inc.

One Harbor Plaza

Stamford, CT 06902

(203) 964-6109

Mr. Kenneth H. Speiser, Manager

Grumman Energy Systems, Inc.

10 Orville, Orive

Bohemia, NY 11716

(516) $244-2786$

Mr. William Stony

Southern Solar Energy Center

61 Perimeter Park

Atlanta, GA 30341

(404) 458-8765

Mr. Thomas Wiggins, Manager

Resin and Catalyst Sales

Vistron Corp.

1653 Midland Building

Cleveland, $\mathrm{OH} 44113$

Mr. Ralph Williams

Polymer Products Department

E. I. DuPont DeNemours \& Co.

Wilmington, DE 19898

(302) 744-8695
Dr. John Andrews

Solar Technology Group

Brookhaven National Laboratory

Building 701

Upton, NY 11973

(516) 345-7726

(FTS) 666-7726

Dr. Philip D. Metz

Solar Technology Group

Brookhaven National Laboratory

Building 701

Upton, NY 11973

(516) 345-3596

(FTS) 666-3596

Mr. William G. Wilhelm

Solar Technology Group

Brookhaven National Laboratory

Building 701

Upton, NY 11973

(516) $345-4708$

(FTS) $666-4708$

Mr. Robert J. Isler

Brookhaven National Laboratory

Building 120

(516) 345-2405

(FTS) 666-2405

Mr. Thomas E. O'Hare

Brookhaven National Laboratory

Building 120

Upton, NY 11973

(516) $345-3428$

(FTS) 666-3428 


\begin{tabular}{lccc} 
Cost Item for a $4^{\prime} \times 8^{\prime}$ Collector & & New Material & Available Material \\
\cline { 1 - 2 } Frame (.10 per ft.) & $\$ 2.40$ & & 2.40 \\
Glazing Film & 3.97 & 8.96 \\
Absorber. 2 polyester & 1.36 & 14.08 \\
2 aluminum foil & 1.79 & 1.79 \\
Absorber coating (.2/sq.ft.) & .64 & .64 \\
Coupling & .40 & .40 \\
Exterior hardware & .50 & .50 \\
Insulation (.10/sq.ft.) & 3.20 & 3.20 \\
Product Labor & 1.10 & 1.10 \\
& & \\
& 15.36 & 33.07 \\
& & 2.30 & 4.96 \\
& 17.66 & 38.00
\end{tabular}


THIN-FILM COLLECTOR EVALUATION QUESTIONNAIRE

BROOKHAVEN NATIONAL LABORATORY

January 13, 1981

Reviewer 
In addressing the first two questions, consider all of the following: suitability of the materials, amenability to low-cost fabrication, marketing and distribution, system life vs cost, as well as any others you think appropriate.

1. What are the strong points of the thin-film collector concept?

2. What are the greatest potential problems or weaknesses associated with the concept?

The following two questions should be addressed in the light of your answers to Questions 1 and 2.

3. Are our economics sound? Consider both the solar system cost goals and the BNL collector manufacturing cost projections.

4. Is this technology sufficiently promising to warrant further effort? Why or why not? 
The remaining questions are appropriate if you feel further development
is warranted. If not, leave them blank.

5. Which of the following actions are most needed to bring this technology into the marketplace? Place a 1 beside the action you feel is most critical, a 2 beside the second most important, etc. If you feel an action is relatively unimportant place no number alongside. Use the space below to elaborate as you feel necessary.

\section{ACTION}

A. Develop improved materials

B. Refine collector design

C. Develop fabrication techniques

D. Solve balance-of-system problems

E. Develop marketing and distribution channels

F. Other (describe)

6. This set of questions is intended to obtain your opinion concerning the proper role of each of the various actors in this program. What should be the role of industry in any further development? What segments of industry are most needed? What role, if any, should BNL play? Is further government funding needed? If so, how much? Is a partnership between BNL and industry desirable? 
MEMORANDUM

тo: Files $(w)$

DATE: January 14,1981

FROM: William E. Stoney

SUBJECT: Trip Report

On January 13, I attended a meeting at Brookhaven National Laboratory (see attached agenda and list of attendees). The meeting was called by Brookhaven to obtain outside opinions on what should be the next steps in their thin-film plastic collector program.

of the invited outside attendees (see list), myself, the two gentlemen from Grumann, and the two from DuPont attended. The format of the meeting followed the agenda. The morning was spent discussing the planning rationale, the technical performance, the construction details, and potential cost reductions of the thin-film plastic collector. This material is contained in the two reports: "Low-Cost Solar Collectors Using Thin-Film Plastics Absorbers and Glazing" and "Thin-Film Flat-Plate Solar Collectors for Low-Cost Manufacture and Installation." In the afternoon the five outside members met alone and discussed and answered the questions (collectively) asked in the attached questionnaire.

The essence of the group's, essentially unanimous, conclusions follows:

- It was agreed that the essential contribution of the Brookhaven effort was the demonstration that a thin-film plastic absorber could be built to produce performance equal to at least the mid-range or better of the conventional designs.

- The various collector configurations and construction details conceived and built by the laboratory were felt to be useful but not unique contributions.

- The committee noted that the major deterrent to the adoption of the plastic collector would be the common perception that plastics are not as good as copper. It was noted that this is a generic problem which any plastic system must overcome, but that therefore, a long term outdoor exposure program should be started immediately because it would always be necessary to convince the skeptics with test data.

- The committee also noted that none of the laboratory tests had included a heat-welded absorber (the configuration tests had been constructed with adhesive binding because of the lack of welding capability at the laboratory). It was therefore recommended that several sets of heat-welded configurations be constructed and tested as quickly as possible.

- The group felt that the cost estimates presented at the mieeting, which range from $55 \$$ per square foot for advanced materials to $\$ 1.19$ per square foot with presently available films, were probably optimistic. Dr. Wilhelm had made these estimates based on a single glazed optimum 
absorption coated design. He used a value of $2 \$$ per square foot for the absorptive coating having received quotes in that range. Conventional techniques cost \$1-2 per square foot and thus, the Grumann representatives registered some skeptism at the low number. In any case, it was. agreed that a production cost of $\$ 5$ per square foot was certainly reasonable with currently available materials and that that was sufficient to be of interest to the industry.

- It was pointed out that the collector itself represented approximately $1 / 3$ the cost of installed hot water systems and thus, a $50 \%$ reduction in the collector cost should result in a 16-20\% savings in the total savings--not revolutionary, but extremely worthwhile.

- It was noted that the plastic absorber required an ambient pressure drain down system configuration. The Grumann representatives noted that this might be considered a problem by some designers especially those who had initially experimented with drain down systems and had had troubles due to freezing.

The above sumarizes the more or less factual findings of the committee. On the crucial question of what next, the committee strongly recommended transferring the good news to industry as rapidly and forcefully as possible. In particular, it was suggested that:

- The feasibility of high performance plastic absorbers should be presented to those segments of industry capable of adapting it as soon as possible. It was pointed out that industry could adapt the concept in a wide variety of configurations going all the way from relatively simple substitution of plastic absorbers for the copper absorber in conventional glass and metal collectors, to the creation of all plastic thin-film configurations which could include simple replacement of the glazing film as a design feature should the use of currently available plastics require that.

- The discussion on transferring the technology to industry brought forth an idea from $\mathrm{Mr}$. Speiser that the laboratory consider producing perhaps as many as fifty or so plastic absorbers that would be handed out to attendees at the technology transfer meeting. He pointed out that the main problem in the adaptation of any new technology involves first convincing the technical staff of a corporation who must in turn have the hard material to convince management to spend hard resources in the development process. He felt that there would be nothing better than a sample which could be tested and displayed back at the home plant. 
FROM: William E. Stoney

- Mr. Speiser noted that it might be useful to combine the regional centers' technology transfer capabilities with the laboratory's R\&D function in developing the approach to disclosure to industry.

- It was finally noted that there were areas, especially in component and total system configuration design which should receive further R\&D by Brookhaven, especially in the development of low-cost drain down systems of the type necessary for use with the plastic absorber.

WES: smb

Attachments (4)

cc: L. Leonaitis

B. Denny

K. Turkia 